\title{
TEORIA DE MODELOS DE CORPOS $p$-ÁDICOS COM FUNÇÕES ANALÍTICAS
}

\section{LEONARDO PRIETO SANABRIA}

TESE APRESENTADA

$\mathrm{AO}$

INSTITUTO DE MATEMÁTICA E ESTATÍSTICA

DA

UNIVERSIDADE DE SÃO PAULO

PARA

OBTENÇÃO DO GRAU DE DOUTOR

$\mathrm{EM}$

MATEMÁTICA

Área de Concentração: Lógica Matemática

Orientador: Prof. Dr. Ricardo Bianconi

Durante a elaboração deste trabalho, o autor recebeu apoio financeiro do Conselho Nacional de Desenvolvimento Científico e Tecnológico - CNPq. 


\title{
TEORIA DE MODELOS DE CORPOS p-ÁDICOS COM FUNÇÕES ANALÍTICAS
}

\author{
LEONARDO PRIETO SANABRIA
}

Este exemplar corresponde à redação final da tese de doutorado, devidamente corrigida, defendida por Leonardo Prieto Sanabria e aprovada pela comissão julgadora.

São Paulo, 5 de Março de 2004.-

Banca examinadora:

- Prof. Dr. Ricardo Bianconi (orientador) - IME-USP

- Prof. Dr. Paulo Agozzini Martin - IME-USP

- Profa. Dra. Itala Maria Loffredo D'Ottaviano - UNICAMP

- Prof. Dr. Marcelo Esteban Coniglio - UNICAMP

- Prof. Dr. Ruy José Guerra Barretto de Queiroz - UFPE 
Dedicada a minha família e à memória de Victor Julio Rodriguez e Javier Enrique Aristizabal 


\section{AGRADECIMENTOS}

Agradeço ao professor Ricardo Bianconi pelo apoio que me deu durante estes quatro anos; suas orientações foram fundamentais na elaboração desta tese.

Aos professores Fernando Zalamea e Leonardo Rendón, e ao meu colega Jairo Eloy, agradeço ter posibilitado o início do meu curso de doutorado.

O exemplo de vida dos meus pais, Eloisa Sanabria Mora e José Prieto Rios, sempre será motivo de meu mais profundo agradecimento. Sou grato também aos meus irmãos Ernesto e Rubiela, a eles devo muito do que eu sou.

Os meus amigos foram a minha família no Brasil; estou especialmente agradecido a Hernan, Mariela, Sandra e Paola.

Também agradeço aos caros colegas Claus, Cristian e Hugo, que deram várias sugestões na escrita desta tese e na preparação da defesa.

A Florencia não só devo sua essencial colaboração na elaboração deste trabalho, como também todos os momentos vividos ao seu lado, para ela todo meu agradecimento e amor. 


\section{RESUMO}

O objetivo principal deste trabalho é estudar algumas propriedades de expansões, por adição de funções analíticas, das estruturas $(I,+, \cdot, 0,1, P)$, e $(\mathcal{D},+, \cdot, 0,1, P)$, onde $I$ denota o disco unitário de $\overline{\mathbb{Q}}_{p}, \mathcal{D}$ o disco unitário de $\mathbb{C}_{p}$, e $P$ é o predicado binário $P(x, y):=|y| \leq|x|$. Primeiro provamos um teorema de eliminação de quantificadores para uma expansão do disco unitário de $\overline{\mathbb{Q}}_{p}$, depois obtemos um resultado análogo para uma expansão de $\mathcal{D}$. A seguir. mostramos algumas conseqüências desses resultados no estudo de conjuntos definíveis.

Nas conclusões formulamos alguns problemas abertos e damos possíveis caminhos para resolvê-los. 


\section{ABSTRACT}

We study expansions of the structures $(I,+, \cdot, 0,1, P)$ and $(\mathcal{D},+, \cdot, 0,1, P)$, by adding analytic functions, where $I$ and $\mathcal{D}$ are the unit discs of $\overline{\mathbb{Q}}_{p}$ (the algebraic closure of $\mathbb{Q}_{p}$ ) and of $\mathbb{C}_{p}$ (its completion), respectively.

We prove the elimination of quantifiers for such expansions and apply these results to the study of definable sets.

We conclude with some open problems and indicate some directions for further work. 


\section{SUMÁRIO}

Capítulo 1. Noções Básicas ..................................... 1

Modelo Completude da Teoria de Corpos Valorados

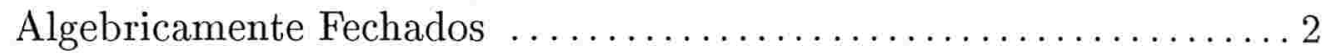

Eliminação de Quantificadores para Corpos Valorados

Algebricamente Fechados com Valoração não Trivial $\ldots \ldots \ldots \ldots . \ldots 21$

Capítulo 2. Um Teorema de Eliminação de Quantificadores

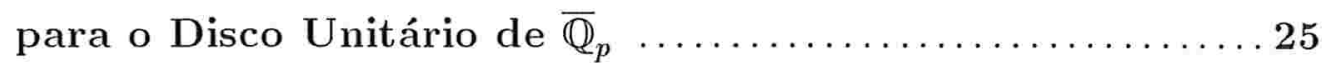

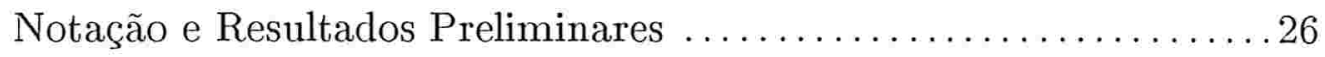

Prova do Teorema Principal Assumindo o Lema Básico ............30

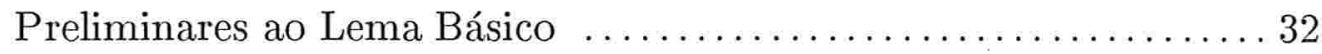

Teorema de Divisão e Preparação de Weierstrass no

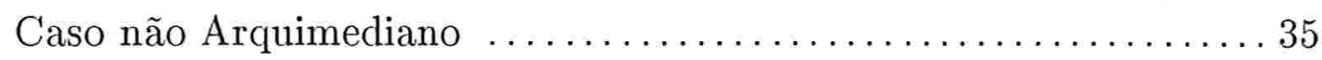

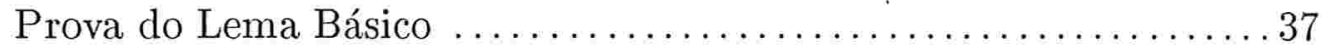

Capítulo 3. Um Teorema de Eliminação de Quantificadores para o Disco Unitário de $\mathbb{C}_{p} \ldots \ldots \ldots \ldots \ldots \ldots \ldots \ldots \ldots \ldots$

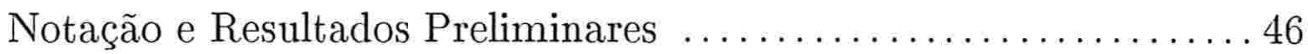

Prova do Teorema Principal $\ldots \ldots \ldots \ldots \ldots \ldots \ldots \ldots \ldots \ldots \ldots \ldots \ldots$ 
Capítulo 4. Conjuntos Semi-algébricos em $\mathbb{C}_{p}$ e $\overline{\mathbb{Q}}_{p}$, Semi-analíticos e Subanalíticos de $\mathbb{C}_{p}$ 49

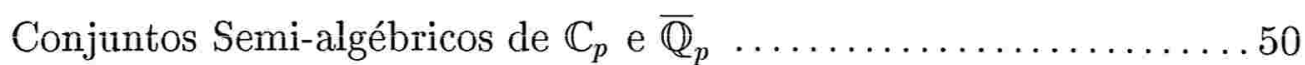

Funções Analíticas p-ádicas Complexas $\ldots \ldots \ldots \ldots \ldots \ldots \ldots \ldots . \ldots \ldots 1$

Variedades Analíticas $p$-ádicas Complexas ................... 53

Conjuntos Subanalíticos de $\mathbb{C}_{p} \ldots \ldots \ldots \ldots \ldots \ldots \ldots \ldots \ldots \ldots$

Capítulo 5. Sistema de Weierstrass em $\overline{\mathbb{Q}}_{p}$ e $\mathbb{C}_{p} \ldots \ldots \ldots \ldots \ldots \ldots \ldots \ldots$

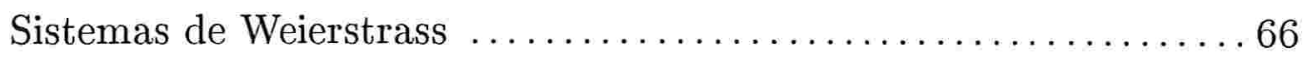

Sistemas de Weierstrass Não Arquimedianos $\ldots \ldots \ldots \ldots \ldots \ldots \ldots . \ldots 70$

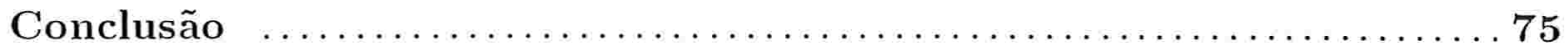

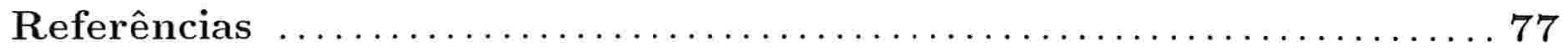




\section{CAPÍTULO 1}

\section{NOÇÕES BÁSICAS}

Apresentamos neste capítulo dois importantes teoremas, que serão usados para obter os resultados desta tese. Na primeira seção, mostramos uma prova da modelo completude da teoria de corpos algebricamente fechados com valoração não trivial, dada por Robinson em [R, (3.4.21)]. Macintyre, McKenna, e van den Dries, usando o trabalho de Robinson, mostram em [M-McK-vdD, (4.2)] que esta mesma teoria possui eliminação de quantificadores; este resultado é dado na segunda seção deste capítulo. 


\section{Modelo Completude da Teoria de Corpos \\ Valorados Algebricamente fechados}

1.1 Axiomatizamos a noção de corpos valorados algebricamente fechados usando as seguintes relações

$$
\begin{array}{ll}
F(x) & x \text { é um elemento do corpo } \\
G(x) & x \text { é um elemento do grupo de valoração } \\
E(x, y) & \text { relação de igualdade. Denotada } x=y \\
V(x, y) & y \text { é a valoração de } x . \text { Denotada por } v(x)=y \\
Q(x, y) \quad \text { ordem do grupo de valoração. Denotado por } x \leq y \\
S^{\prime}(x, y, z) \text { adição do grupo de valoração. Denotada por } x \oplus y=z \\
S(x, y, z) \quad \text { adição do corpo. Denotada por } x+y=z \\
P(x, y, z) \quad \text { produto do corpo. Denotado por } x y=z
\end{array}
$$

A caracterização dos corpos valorados algebricamente fechados é dada pela união dos conjuntos de axiomas apresentados nos seguintes itens 1.2 a 1.7.

1.2 Axiomas para a igualdade

$$
\begin{aligned}
& \forall x(x=x) \\
& \forall x y(x=y \rightarrow y=x) \\
& \forall x y z(x=y \wedge y=z \rightarrow x=z) \\
& \forall x_{1} \cdots x_{n} \forall y_{1} \cdots y_{n}\left(R\left(x_{1}, \ldots, x_{n}\right) \wedge x_{1}=y_{1} \wedge \cdots \wedge x_{n}=y_{n} \rightarrow R\left(y_{1}, \ldots, y_{n}\right)\right)
\end{aligned}
$$

onde $R \in\left\{F, G, V, Q, S^{\prime}, S, P\right\}$ dado em 1.1. 
1.3 Axiomas para corpo

$$
\begin{aligned}
& \forall x y \exists z(F(x) \wedge F(y) \rightarrow F(z) \wedge x+y=z) \\
& \forall x y \exists z(F(x) \wedge F(y) \rightarrow F(z) \wedge x y=z) \\
& \forall x y z w(F(x) \wedge F(y) \wedge F(z) \wedge F(w) \rightarrow(x+y=z \wedge x+y=w \rightarrow z=w)) \\
& \forall x y z w(F(x) \wedge F(y) \wedge F(z) \wedge F(w) \rightarrow(x y=z \wedge x y=w \rightarrow z=w)) \\
& \forall u v w x y z(F(u) \wedge F(v) \wedge F(w) \wedge F(x) \wedge F(y) \wedge F(z) \rightarrow \\
& \forall u v w x y z(F(u) \wedge F(v) \wedge F(w) \wedge F(x) \wedge F(y) \wedge F(z) \rightarrow \\
& \qquad(u v=w \wedge w x=y \wedge v x=z \rightarrow u z=y)) \\
& \forall x y z(F(x) \wedge F(y) \wedge F(z) \rightarrow(x+y=z \rightarrow y+x=z)) \\
& \forall x y z(F(x) \wedge F(y) \wedge F(z) \rightarrow(x y=z \rightarrow y x=z)) \\
& \forall t u v w x y z(F(t) \wedge F(u) \wedge F(v) \wedge F(w) \wedge F(x) \wedge F(y) \wedge F(z) \rightarrow \\
& \qquad(t u=v \wedge w u=x \wedge v+x=y \wedge t+w=z \rightarrow z u=y)) \\
& \forall x y \exists z(F(x) \wedge F(y) \rightarrow F(z) \wedge x+z=y) \\
& \forall x y \exists z(F(x) \wedge F(y) \rightarrow F(z) \wedge(x+x=x \vee x z=y) \\
& \exists x y(F(x) \wedge F(y) \wedge \neg(x=y))
\end{aligned}
$$

1.4 Axiomas de grupo abeliano ordenado

$$
\begin{aligned}
& \forall x y \exists z(G(x) \wedge G(y) \rightarrow G(z) \wedge x \oplus y=z) \\
& \forall x y z w(G(x) \wedge G(y) \wedge G(z) \wedge G(w) \rightarrow(x \oplus y=z \wedge x \oplus y=w \rightarrow z=w)) \\
& \forall u v w x y z(G(u) \wedge G(v) \wedge G(w) \wedge G(x) \wedge G(y) \wedge G(z) \rightarrow \\
& \qquad(u \oplus v=w \wedge w \oplus x=y \wedge v \oplus x=z \rightarrow u \oplus z=y)) \\
& \forall x y z(G(x) \wedge G(y) \wedge G(z) \rightarrow(x \oplus y=z \rightarrow y \oplus x=z))
\end{aligned}
$$




$$
\begin{aligned}
& \forall x y \exists z(G(x) \wedge G(y) \rightarrow G(z) \wedge x \oplus z=y) \\
& \exists x y(G(x) \wedge G(y) \wedge \neg(x=y)) \\
& \forall x y z(G(x) \wedge G(y) \wedge G(z) \rightarrow(x \leq y \wedge y \leq z \rightarrow x \leq z)) \\
& \forall x y(G(x) \wedge G(y) \rightarrow x \leq y \vee y \leq x) \\
& \forall x y(G(x) \wedge G(y) \rightarrow((x \leq y \wedge y \leq x) \equiv x=y)) \\
& \forall x y z v w(G(x) \wedge G(y) \wedge G(z) \wedge G(v) \wedge G(w) \rightarrow
\end{aligned}
$$$$
(x \oplus y=z \wedge x \oplus v=w \wedge y \leq v \rightarrow z \leq w))
$$

1.5 Os seguintes axiomas estabelecem que em qualquer modelo, os conjuntos de constantes que satisfazem $F(x)$ e $G(x)$, respectivamente, constituem conjuntos complementares, tais que as relações $S$ e $P$ são válidas só no primeiro, enquanto as relações $Q, S^{\prime}$ são satisfeitas só por elementos do segundo.

$$
\begin{aligned}
& \forall x((F(x) \vee G(x)) \wedge(\neg F(x) \vee \neg G(x))) \\
& \forall x y(x \leq y \rightarrow G(x) \wedge G(y)) \\
& \forall x y z(x \oplus y=z \rightarrow G(x) \wedge G(y) \wedge G(z)) \\
& \forall x y z(x+y=z \rightarrow F(x) \wedge F(y) \wedge F(z)) \\
& \forall x y z(x y=z \rightarrow F(x) \wedge F(y) \wedge F(z))
\end{aligned}
$$

1.6 Propriedades da valoração

$$
\begin{aligned}
& \forall x y(v(x)=y \rightarrow F(x) \wedge G(y) \wedge \neg(x+x=x)) \\
& \forall x \exists y(F(x) \rightarrow(x+x=x \vee(G(y) \wedge v(x)=y))) \\
& \forall x \exists y(G(x) \rightarrow v(y)=x) \\
& \forall x y z(v(x)=y \wedge v(x)=z \rightarrow y=z) \\
& \forall u v w x y z(v(u)=x \wedge v(v)=y \wedge v(w)=z \wedge u v=w \rightarrow x \oplus y=z) \\
& \forall u v w x y z(v(u)=x \wedge v(v)=y \wedge v(w)=z \wedge u+v=w \wedge x \leq y \rightarrow x \leq z)
\end{aligned}
$$


Os axiomas dados em 1.2 até 1.6 caracterizam o conceito de corpo valorado, onde o grupo de valoração é um grupo abeliano ordenado (totalmente). Para o zero do corpo não é dada uma valoração, mas aceitamos a convenção

$$
v(0)=\infty
$$

e agora para todas $x, y \in F$ temos

$$
v(x y)=v(x)+v(y)
$$

(no que se segue usaremos + em lugar de $\oplus$ ) e

$$
v(x+y) \geq \min \{v(x), v(y)\}
$$

Agora precisamos acrescentar os axiomas que estabelecem que o corpo é algebricamente fechado. Primeiro definimos as relações

$$
\begin{aligned}
& P_{0}(x, y)=(x=x \wedge \neg(y+y=y) \wedge(y y=y)) \\
& P_{n}(x, y)=\left(\exists z\left(P_{n-1}(x, z) \wedge z x=y\right)\right) \quad n=1,2, \ldots
\end{aligned}
$$

assim, $P_{n}(x, y)$ expressa $y=x^{n}$. Agora para $n \geq 1$ definimos

$$
\begin{aligned}
& R_{n}\left(x_{0}, x_{1}, \ldots, x_{n}, y\right):=\exists u_{1} \cdots u_{n} \exists v_{1} \cdots v_{n} \exists w_{2} \cdots w_{n-1}\left(P_{1}\left(y, u_{1}\right) \wedge \cdots \wedge P_{n}\left(y, u_{n}\right)\right. \\
& \wedge x_{1} u_{1}=v_{1} \wedge \cdots \wedge x_{n} u_{n}=v_{n} \wedge v_{1}+v_{2}=w_{2} \\
&\left.\wedge w_{2}+v_{3}=w_{3} \wedge \cdots \wedge w_{n-1}+v_{n}=x_{0}\right)
\end{aligned}
$$

que significa (salvo modificações óbvias para $n=1,2$ )

$$
x_{n} y^{n}+x_{n-1} y^{n-1}+\cdots+x_{2} y^{2}+x_{1} y=x_{0} .
$$


1.7 Os seguintes axiomas estabelecem que o corpo é algebricamente fechado.

$$
\forall x_{0} \cdots x_{n} \exists y\left(F\left(x_{0}\right) \wedge \cdots \wedge F\left(x_{n}\right) \rightarrow F(y) \wedge\left(x_{n}+x_{n}=x_{n} \vee R_{n}\left(x_{0}, \ldots, x_{n}, y\right)\right)\right),
$$

para $n \geq 2$.

Seja $K$ a coleção de axiomas apresentados em 1.2-1.7. Então, todo modelo $M$ de $K$ compreende um grupo $G$ e um corpo $F$. Queremos provar nesta seção que $K$ é modelo completa.

1.8 Seja $M$ um modelo de $K$. Vejamos que então o grupo $G$ é completamente divisível.

Dada $a \in G$ e $n$ inteiro positivo, a equação

$$
n x=a
$$

possui solução em $G$. De fato, existe um elemento $b \in F$ tal que $a=v(b)$. Seja $c \in F$ tal que $c^{n}=b$. Então se $x=v(c)$ temos

$$
n x=n v(c)=v\left(c^{n}\right)=v(b)=a .
$$

1.9 Seja $F$ um corpo algebricamente fechado o qual é valorado em um grupo $G$, e seja $F(t)$ uma extensão transcendental simples de $F$.

Suponha que existem duas valorações $v_{1}, v_{2}$ de $F(t)$ tais que para todo $a$ em $F$,

$$
v_{1}(a)=v_{2}(a) \text { e } v_{1}(t-a)=v_{2}(t-a) .
$$

Então

$$
v_{1}(\tau)=v_{2}(\tau)
$$

para todo $\tau \neq 0$ em $F(t)$. 
De fato, cada $\tau \neq 0$ de $F(t)$ pode-se escrever como uma função racional em $t$ com coeficientes em $F$,

$$
\tau=\frac{p(t)}{q(t)}=a \frac{\left(t-a_{1}\right) \cdots\left(t-a_{k}\right)}{\left(t-b_{1}\right) \cdots\left(t-b_{l}\right)}, \quad a, a_{1}, \ldots, a_{k}, b_{1}, \ldots, b_{l} \in F
$$

onde $a \neq 0$, e o numerador (e/ou denominador) pode-se reduzir à unidade. Então

$$
\begin{aligned}
v_{1}(\tau) & =v_{1}(a)+v_{1}\left(t-a_{1}\right)+\cdots+v_{1}\left(t-a_{k}\right)-v_{1}\left(t-b_{1}\right)-\cdots-v_{1}\left(t-b_{l}\right) \\
& =v_{2}(a)+v_{2}\left(t-a_{1}\right)+\cdots+v_{2}\left(t-a_{k}\right)-v_{2}\left(t-b_{1}\right)-\cdots-v_{2}\left(t-b_{l}\right) \\
& =v_{2}(\tau) .
\end{aligned}
$$

1.10 Seja $F^{\prime}$ um corpo com grupo de valoração $G^{\prime}$, e seja $F$ um subcorpo de $F^{\prime}$, tal que $F^{\prime}$ é de grau de transcendência 1 sobre $F$. Seja $G$ o grupo de valoração de $F$, $G \subseteq G^{\prime}$. Então dados $b_{1}, b_{2} \in G^{\prime}$, existem inteiros $n_{1}, n_{2}$, pelo menos um diferente de zero, tais que

$$
n_{1} b_{1}+n_{2} b_{2} \in G \text {. }
$$

Para provar isto usamos que se $v(x) \neq v(y)$ então

$$
v(x+y)=\operatorname{mín}\{v(x), v(y)\}
$$

Dados $b_{1}, b_{2} \in G^{\prime}$, podemos encontrar $t_{1}, t_{2} \in F^{\prime}$, tais que

$$
v\left(t_{1}\right)=b_{1}, \quad v\left(t_{2}\right)=b_{2} .
$$

Já que $F^{\prime}$ é de grau de transcendência 1 sobre $F$, existe uma relação algébrica

$$
\sum a_{k m} t_{1}^{k} t_{2}^{m}=0 \quad\left(a_{k m} \in F\right)
$$


tal que, pelo menos um $a_{k m}, k+m>0$, é não nulo (portanto existem ao menos dois $a_{k m}$ não nulos). Omitimos os termos $a_{k m}=0$. Então

$$
v\left(a_{k m} t_{1}^{k} t_{2}^{m}\right)=v\left(a_{k m}\right)+k b_{1}+m b_{2}
$$

Consideremos dois termos diferentes, $a_{k m} t_{1}^{k} t_{2}^{m}$ e $a_{k^{\prime} m^{\prime}} t_{1}^{k^{\prime}} t_{2}^{m^{\prime}}$, em (3). Então

$$
v\left(a_{k m} t_{1}^{k} t_{2}^{m}\right)=v\left(a_{k^{\prime} m^{\prime}} t_{1}^{k^{\prime}} t_{2}^{m^{\prime}}\right)
$$

é equivalente a

$$
v\left(a_{k m}\right)+k b_{1}+m b_{2}=v\left(a_{k^{\prime} m^{\prime}}\right)+k^{\prime} b_{1}+m^{\prime} b_{2}
$$

Ou

$$
\left(k-k^{\prime}\right) b_{1}+\left(m-m^{\prime}\right) b_{2}=v\left(a_{k^{\prime} m^{\prime}}\right)-v\left(a_{k m}\right) \in G .
$$

Já que assumimos que estamos tratando com dois termos diferentes de (3), temos $k-k^{\prime} \neq 0$ ou $m-m^{\prime} \neq 0$ (ou ambos são diferentes de zero) e, desta maneira, obteríamos (1).

Suponha que (4) não é satisfeita para nenhum par de termos diferentes de (3). Então repetindo sucessivamente (2), temos que

$$
v\left(\sum a_{k m} t_{1}^{k} t_{2}^{m}\right)=\min \left\{v\left(a_{k m} t_{1}^{k} t_{2}^{m}\right)\right\}
$$

mas isto implica que $v\left(\sum a_{k m} t_{1}^{k} t_{2}^{m}\right)$ é finita (isto é, um elemento próprio do grupo de valoração), contradizendo

$$
v\left(\sum a_{k m} t_{1}^{k} t_{2}^{m}\right)=v(0)=\infty
$$

Isto prova (1).

1.12 Agora seja $F$ um corpo algebricamente fechado, o qual é valorado em um grupo $G$ e seja $F(t)$ uma extensão simples de $F$ a qual é valorada em uma extensão $G^{\prime}$ de $G$, 
tal que a valoração de $F(t)$ é uma extensão da valoração de $F$. Suponha que $b=v(t)$ não está em $G$, então a valoração de $F(t)$ em $G^{\prime}$ está determinada pelas relações dadas por

$$
b<d \quad \text { e } \quad b>d
$$

as quais valem em $G^{\prime}$, para todos os elementos $d \in G$.

De fato, para todo $a$ de $F$,

$$
v(t-a)=\operatorname{mín}\{v(t), v(a)\}=\operatorname{mín}\{b, v(a)\},
$$

já que $v(t)=b \neq v(a)$. Assim, o valor funcional de $v(t-a)$ é determinado inteiramente pelo valor $v(a)$ em $G$, pelo conjunto de relações (1). Agora, cada elemento $\tau \neq 0 \in$ $F(t)$ pode-se escrever como

$$
\tau=a \frac{\left(t-a_{1}\right) \cdots\left(t-a_{k}\right)}{\left(t-b_{1}\right) \cdots\left(t-b_{l}\right)} \quad a, a_{1}, \ldots, a_{k}, b_{1}, \ldots, b_{l} \in F
$$

portanto

$$
v(\tau)=v(a)+v\left(t-a_{1}\right)+\cdots+v\left(t-a_{k}\right)-v\left(t-b_{1}\right)-\cdots-v\left(t-b_{l}\right),
$$

e, em particular, para cada inteiro $n$, temos

$$
v\left(t^{n}\right)=n v(t)=n b
$$

Segue que $G^{\prime}$ consiste de todos os elementos da forma

$$
d+n b, d \in G, \quad n \in \mathbb{Z} .
$$

Esta representação é única, já que $G^{\prime}$ é ordenado e $G$ é completamente divisível. 
Teorema 1.13. Seja $F$ um corpo valorado em um grupo $G$, e seja $F^{\prime}$ uma extensão algébrica finita normal de F. Sejam $G_{1}^{\prime}$ e $G_{2}^{\prime}$ duas extensões de $G$, em cada uma das quais existe uma valoração $v_{i}, i=1,2$, de $F^{\prime}$ a qual é uma extensão da valoração de $F$. Então existe um automorfismo $f$ de $F^{\prime}$ que centraliza os elementos de $F$, e um isomorfismo h que preserva a ordem entre $G_{1}^{\prime}$ e $G_{2}^{\prime}$, e que centraliza os elementos de $G$ tal que o seguinte diagrama

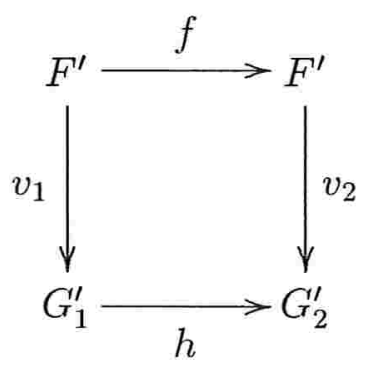

comuta.

Ver a prova deste teorema em $[K,(\S 11$, Tma.19)].

1.14 Seja $F$ um corpo valorado em um grupo $G$, e seja $\bar{F}$ o fecho algébrico de $F$. Sejam $\bar{G}_{1}$ e $\bar{G}_{2}$ duas extensões de $G$ nas quais $\bar{F}$ é valorado, como no Teorema 1.13. Então a conclusão do Teorema 1.13 também vale.

Prova. Considere a coleção de pares $\{(f, h)\}$ que fazem comutar o diagrama

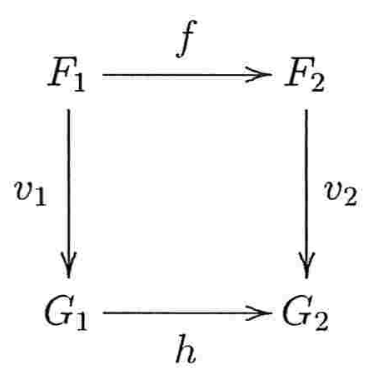

onde os $F_{i}$ são extensões de $F, f$ é um isomorfismo que centraliza $F$, os $G_{i}$ são extensões de $G$, e $h$ é um isomorfismo que preserva a ordem e centraliza $G$. 
O conjunto dos pares $(f, h)$ está ordenado parcialmente por: $(f, h) \preceq\left(f^{\prime}, h^{\prime}\right)$, se $f^{\prime}$ é uma extensão de $f$ e $h^{\prime}$ é uma extensão de $h$. Podemos usar o Lema de Zorn para mostrar que $\{(f, h)\}$ tem um elemento maximal $\left(f^{0}, h^{0}\right)$. Sejam $F_{1}=F_{1}^{0}$, e $F_{2}=F_{2}^{0}$, os corpos correspondentes, e $G_{1}=G_{1}^{0}, G_{2}=G_{2}^{0}$ os grupos de valoração correspondentes. Queremos provar que $F_{1}^{0}=F_{2}^{0}=\bar{F}$. Suponha, pelo contrário, que $F_{1}^{0}$ é um subcorpo próprio de $\bar{F}$; portanto o mesmo se aplica a $F_{2}^{0}$. Seja $F_{1}^{*}$ tal que $\left[F_{1}^{*}: F_{1}^{0}\right]<\infty$, e seja $F_{2}^{*}$ a correspondente extensão de $F_{2}^{0}$, isto é, $F_{1}^{*}$ e $F_{2}^{*}$ são obtidas a partir de $F_{1}^{0}, F_{2}^{0}$ pela adjunção das raízes de correspondentes equações normais. Sejam $G_{1}^{*}$ e $G_{2}^{*}$ os correspondentes grupos de valoração contidos em $\bar{G}_{1}$ e $\bar{G}_{2}$, respectivamente. Então $G_{1}^{0} \subseteq G_{1}^{*}, G_{2}^{0} \subseteq G_{2}^{*}$. Por definição de $\left(f^{0}, h^{0}\right), G_{1}^{0}$ e $G_{2}^{0}$ são isomorfos por um isomorfismo que preserva a ordem. Aplicando 1.13 podemos estender $\left(f^{0}, h^{0}\right)$ a $\left(f^{*}, h^{*}\right)$ para o qual $F_{1}=F_{1}^{*}, F_{2}=F_{2}^{*}, G_{1}^{\prime}=G_{1}^{*}, G_{2}^{\prime}=G_{2}^{*}$. Isto contradiz que $\left(f^{0}, h^{0}\right)$ seja maximal, e mostra que $F_{1}^{0}=\bar{F}$. Consequentemente $F_{2}^{0}=\bar{F}, G_{1}^{0}=\bar{G}_{1}, G_{2}^{0}=\bar{G}_{2}$. Assim $\left(f^{0}, h^{0}\right)$ contém um automorfismo $f^{0}$ de $\bar{F}$ que centraliza $F$ e um isomorfismo $h^{0}$ entre $\bar{G}_{1}$ e $\bar{G}_{2}$ o qual centraliza $G$, tal que

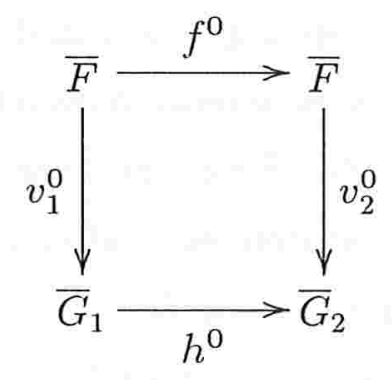

comuta

Teorema 1.15. A teoria elementar de um corpo algebricamente fechado, com valoração não trivial em um grupo abeliano ordenado, é modelo completa.

Prova. Temos que provar que $K$ (a união de 1.2 a 1.7 ) satisfaz: para cada par de modelos $M$ e $M^{\prime}$ de $K$, tais que $M^{\prime}$ é uma extensão de $M$, cada sentença primitiva $\psi$ 
que está definida em $M$ é válida em $M^{\prime}$ se, e só se, é válida em $M$. Lembramos que $\psi$ é primitiva se $\psi$ é da forma

$$
\exists y_{1} \cdots \exists y_{n} \phi\left(y_{1}, \ldots, y_{n}\right)
$$

onde $\phi$ é uma fórmula livre de quantificadores.

Seja $M$ um modelo de $K$ e seja

$$
\psi=\exists y_{1} \cdots \exists y_{n} \phi\left(y_{1}, \ldots, y_{n}\right)
$$

uma sentença primitiva a qual está definida em $K \cup N$, onde $N$ é o diagrama de $M$, tal que $\psi$ é válida em uma extensão $M^{\prime}$ de $M$, a qual é um modelo de $K$. $M$ consiste de um corpo algebricamente fechado $F$ e seu grupo de valoração $G$, e $M^{\prime}$ consiste de uma extensão algebricamente fechada $F^{\prime}$ de $F$ e seu grupo de valoração $G^{\prime}$ é uma extensão de $G$. Se $F=F^{\prime}$, então $G=G^{\prime}$ e $M=M^{\prime}$, e não teríamos nada a provar.

Suponha então que $F$ é um subcorpo próprio de $F^{\prime}$, e que $\psi$ não é válida em $M$ mas vale em $M^{\prime}$. Podemos supor que o grau de transcendência de $F^{\prime}$ sobre $F$ é 1 .

Agora, consideremos separadamente duas alternativas: (i) $G=G^{\prime}$ e (ii) $G \neq G^{\prime}$. Suponha primeiro que $G=G^{\prime}$. Seja $c$ um elemento de $F^{\prime}-F$, e seja $K^{*}$ a união de $K$ e o seguinte conjunto de sentenças (dado em (2) a (4)):

(2) O diagrama de $M$, denotado por $N$.

(3) O conjunto de sentenças $\neg(a=c)$, onde $a$ varia sobre todas as constantes de $M$.

(4) Para cada $a \in F$, se

$$
v(c-a)=b, \quad b \in G
$$

então incluímos a sentença

$$
T(a, b, c)=\exists x(a+x=c \wedge v(x)=b) .
$$

Então o conjunto $K^{*}$ é consistente, já que $M^{\prime}$ é um modelo de $K^{*}$. 
Seja $M^{*}$ um outro modelo de $K^{*} ; M^{*}$ é portanto um modelo de $K$ e contém um corpo algebricamente fechado $F^{*} \supset F$ e um grupo de valoração $G^{*}$ o qual é uma extensão de $G$. Além disso, $F^{*}$ tem que ser uma extensão própria de $F$. De fato, por

$$
\forall x y z\left(x+y=z \rightarrow F^{*}(x) \wedge F^{*}(y) \wedge F^{*}(z)\right)
$$

e por uma das sentenças de (4)

$$
T(a, b, c)=\exists x(a+x=c \wedge v(x)=b),
$$

tem-se que $F^{*}(c)$. E por (3) $c$ é diferente de todos os elementos de $F$.

Já que $F$ é algebricamente fechado, $c$ tem que ser algebricamente independente de $F$. Segue que $F^{*}$ inclui um subcorpo $\widehat{F}$, tal que $c \in \widehat{F}$, o qual é isomorfo a $F^{\prime}$ sob um isomorfismo que centraliza os elementos de $F$ e a constante $c$ (dimensão transcendental de $F^{\prime}$ sobre $F$ é 1 ). Os corpos obtidos ao agregar $c$ a $F$ em $F^{\prime}$ e $\widehat{F}$ são certamente isomorfos, mas a priori não necessariamente coincidem, no entanto podemos, sem perda de generalidade, identificar os elementos correspondentes. Podemos falar então do corpo $F(c)$ como estando contido em $F^{\prime}$ e $\widehat{F}$. Seja $\widehat{G}$ o grupo de valoração de $\widehat{F}$, $\widehat{G} \subseteq G^{*}$, e seja $G_{0}$ o grupo de valoração de $F(c)$, considerado como subcorpo de $\widehat{F}$, $G_{0} \subseteq \widehat{G}$. O grupo de valoração de $F(c)$ considerado como subcorpo de $F^{\prime}$ é $G$ (pela hipótese $\left.G=G^{\prime}\right)$.

Além disso, por (4), a valoração de cada elemento $c-a$ de $F(c)$ em $G_{0}$, onde $a \in F$, coincide com a valoração do mesmo elemento em $G=G^{\prime}$. Aplicando 1.9, temos que $G_{0}$ é isomorfo a $G$. E, por 1.14 , concluímos que $\widehat{G}$ é isomorfo a $G$ por um isomorfismo $h$ que preserva a ordem e que faz comutar o diagrama

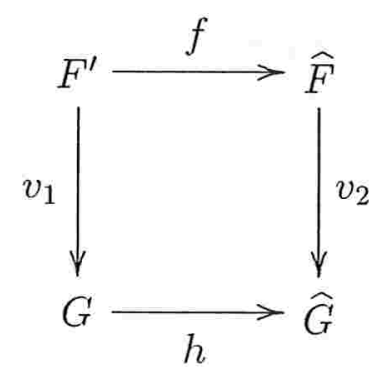


onde $f$ é um isomorfismo entre $F^{\prime}$ e $\widehat{F}$ (o qual centraliza os elementos de $F(c)$ ), e as $v_{i}$ são as valorações correspondentes. O corpo $\widehat{F}$ junto com seu grupo de valoração $\widehat{G}$ constituen um modelo $\widehat{M}$ de $K$, o qual é isomorfo a $M^{\prime}$ pelas transformações $f, h$. com respeito a todas as relações definidas em $M^{\prime}$ e $\widehat{M}$.

Agora, pela hipótese, $\psi$ é válida em $M^{\prime}$. Segue que existem constantes $a_{1}^{\prime}, \ldots, a_{n}^{\prime}$, as quais podem estar parte em $F^{\prime}$ e parte em $G^{\prime}=G$, tais que $\phi\left(a_{1}^{\prime}, \ldots, a_{n}^{\prime}\right)$ vale em $M^{\prime}$. Sejam $\widehat{a}_{1}, \ldots, \widehat{a}_{n}$ as correspondentes constantes de $\widehat{M}$ pelo isomorfismo $f$ (i.e. $\left.f\left(a_{i}^{\prime}\right)=\widehat{a}_{i}\right)$. Então $\phi\left(\widehat{a}_{1}, \ldots, \widehat{a}_{n}\right)$ é válida em $\widehat{M}$ e, já que esta fórmula não inclui quantificadores, também é válida em $M^{*}$.

Mas isto mostra que $\psi$ é válida em $M^{*}$, i.e, $\psi$ vale em qualquer modelo de $K^{*}$. Segue que $\psi$ é dedutível de $K^{*}$. Isto quer dizer, por (3) e (4), que existe um número finito de elementos de $F$,

$$
a_{1}, \ldots, a_{j}, a_{j+1}, \ldots, a_{l}
$$

e um número finito de elementos de $G$

$$
b_{j+1}, \ldots, b_{l}
$$

tais que a sentença

$$
\left(\neg\left(a_{1}=c\right) \wedge \cdots \wedge \neg\left(a_{j}=c\right) \wedge T\left(a_{j+1}, b_{j+1}, c\right) \wedge \cdots \wedge T\left(a_{l}, b_{l}, c\right)\right) \rightarrow \psi
$$

é dedutível de $K \cup N$. Mas $c$ não está incluída em $K \cup N$ e portanto (5) implica que

$$
\exists x\left(\neg\left(a_{1}=x\right) \wedge \cdots \wedge \neg\left(a_{j}=x\right) \wedge T\left(a_{j+1}, b_{j+1}, x\right) \wedge \cdots \wedge T\left(a_{l}, b_{l}, x\right)\right) \rightarrow \psi
$$

é dedutível de $K \cup N$. Para mostrar que $\psi$ vale em $M$ (o qual é um modelo de $K \cup N$ ) basta provar então que

$$
\exists x\left(\neg\left(a_{1}=x\right) \wedge \cdots \wedge \neg\left(a_{j}=x\right) \wedge T\left(a_{j+1}, b_{j+1}, x\right) \wedge \cdots \wedge T\left(a_{l}, b_{l}, x\right)\right)
$$


vale em $M$. Portanto precisamos provar que o conjunto de condições

$$
\begin{array}{ll}
x \neq a_{i} & i=1, \ldots, j \\
v\left(x-a_{i}\right)=b_{i} & i=j+1, \ldots, l
\end{array}
$$

possui uma solução em $M$, para $a_{i} \in F, b_{i} \in G$, onde é conhecido que (6) possui uma solução $x=c$ em uma extensão $M^{\prime}$ de $M \operatorname{com} F \subseteq F^{\prime}$ e $G \subseteq G^{\prime}$. Agora, já que $c \neq a_{i}, i=1, \ldots, j$, segue que $v\left(c-a_{i}\right)$ possui um valor $b_{i}$ em $G$, e, reciprocamente, se $v\left(x-a_{i}\right)=b_{i} \in G$, então $x \neq a_{i}$. Portanto podemos substituir (6) por um sistema simples

$$
v\left(x-a_{i}\right)=b_{i}, \quad i=1, \ldots, l .
$$

Agora, se $a_{i}=a_{k}$ para $i \neq k$ então também temos que $b_{i}=b_{k}$, já que (7) tem uma solução em $M^{\prime}$. Assim as duas equações coincidem, i.e. podemos assumir $a_{i} \neq a_{k}$ para $i \neq k$. Agora consideremos um par de equações em $(7)$ tais que $b_{i} \neq b_{k}$. Então $v\left(a_{i}-a_{k}\right)=v\left(a_{k}-a_{i}\right)$ tem que ser diferente de pelo menos uma das duas constantes $b_{i}, b_{k}$. Suponha $v\left(a_{i}-a_{k}\right) \neq b_{i}$. Então

$$
v\left(x-a_{k}\right)=v\left(x-a_{i}+a_{i}-a_{k}\right)=\min \left\{b_{i}, v\left(a_{i}-a_{k}\right)\right\} .
$$

Isto quer dizer, para cada $x$ que satisfaz

$$
v\left(x-a_{i}\right)=b_{i}
$$

o valor de $v\left(x-a_{k}\right)$ é determinado realmente pela valoração de $F$. Portanto podemos omitir a equação

$$
v\left(x-a_{k}\right)=b_{k}
$$

em (7). Repetindo este procedimento, se é necessário, finalmente chegamos ao sistema de condições

$$
v\left(x-a_{i}\right)=b \quad i=1, \ldots, m
$$


com o mesmo $b$ para todas as equações.

Agora assumamos que $v\left(a_{1}-a_{i}\right) \neq b$ para algum $i \geq 2$. Então

$$
b=v\left(x-a_{i}\right)=v\left(x-a_{1}+a_{1}-a_{i}\right)=\operatorname{mín}\left\{b, v\left(a_{1}-a_{i}\right)\right\}
$$

e portanto temos que $v\left(a_{1}-a_{i}\right)>b$. Assim, cada $x$ que satisfaz $v\left(x-a_{1}\right)=b$, satisfaz também $v\left(x-a_{i}\right)=b$. Então podemos omitir a correspondente condição (8), e podemos assumir que

$$
v\left(a_{1}-a_{i}\right)=b \quad \text { para } i=2,3, \ldots m \text {. }
$$

Escolhemos um elemento $a_{0} \in F$ tal que $v\left(a_{0}\right)=b$, e fazemos

$$
y=\frac{x-a_{1}}{a_{0}} .
$$

Então a primeira condição em (8) implica

$$
v(y)=v\left(x-a_{1}\right)-v\left(a_{0}\right)=b-b=0 .
$$

Além disso, fazendo $c_{i}=\left(a_{i}-a_{1}\right) / a_{0}$ para $i=2, \ldots, m$ obtemos de (8) que

$$
v\left(y-c_{i}\right)=v\left(\frac{x-a_{1}-\left(a_{i}-a_{1}\right)}{a_{0}}\right)=v\left(\frac{x-a_{i}}{a_{0}}\right)=b-b=0 \quad(i=2, \ldots, m) .
$$

Portanto pode-se substituir (8) por

$$
\begin{aligned}
& v(y)=0 \\
& v\left(y-c_{i}\right)=0 \quad i=2, \ldots, m
\end{aligned}
$$

onde $v\left(c_{i}\right)=v\left(a_{i}-a_{1}\right)-v\left(a_{0}\right)=b-b=0, c_{i} \in F$.

(10) é satisfeito em $M^{\prime}$ por $y=\left(c-a_{1}\right) / a_{0}$, e temos que resolvê-la em $M$. Cada solução de (10) gera uma solução de (8), tomando $x=a_{0} y+a_{1}$. 
Seja $R$ o anel de valoração de $F$, i.e, o conjunto de todos os $r \in F$ para os quais $v(r) \geq 0$ (incluído o zero do corpo), e seja $J$ o ideal da valoração, i.e. o conjunto de todos os elementos $r \in F$ tais que $v(r)>0$. Então o quociênte $F_{R}=R / J$ é um corpo. Os elementos de $F_{R}$ são as classes disjuntas dos elementos de $R$, e denotamos por $\gamma_{2}, \ldots, \gamma_{m}$ os elementos de $F_{R}$ os quais contêm $c_{2}, \ldots, c_{m}$ respectivamente. Então $\gamma_{i} \neq 0$, i.e. $\gamma_{i}$ não está em $J, i=2, \ldots, m$, já que $v\left(c_{i}\right)=0$. Consideremos o sistema de inequações

$$
\begin{aligned}
& \eta \neq 0 \\
& \eta \neq \gamma_{i} \quad i=2, \ldots, m .
\end{aligned}
$$

Para provar que este sistema possui uma solução em $F_{R}$, é suficiente provar que $F_{R}$ é infinito. Isto é claro quando $F_{R}$ é de característica 0 . Se $F_{R}$ é de característica $p>0$, seja $q>0$ um inteiro não divisível por $p$, e sejam $e_{1}, \ldots, e_{q}$ as raízes da equação

$$
Z^{q}-1=0 .
$$

Então $v\left(e_{i}\right)=0$, já que $0=v(1)=v\left(e_{i}^{q}\right)=q v\left(e_{i}\right)$. Portanto $e_{i}$ está em um elemento $\xi_{i}$ de $F_{R}$. Além disso, a identidade

$$
Z^{q}-1=\left(Z-e_{1}\right) \cdots\left(Z-e_{q}\right) \quad \text { em } F
$$

implica

$$
Z^{q}-1=\left(Z-\xi_{1}\right) \cdots\left(Z-\xi_{q}\right) \quad \text { em } F_{R}
$$

i.e. $\xi_{1}, \ldots, \xi_{q}$ são as $q$-ésimas raízes da unidade em $F_{R}, \xi_{i} \neq \xi_{k}$, para $i \neq k$. Já que $q$ pode ser tão grande quanto se queira, $F_{R}$ é infinito.

Agora seja $\eta=\alpha \in F_{R}$ uma solução de (11) e seja $a$ um elemento de $\alpha$. Então $a \in R$, e $a \notin J$ e $a-c_{i} \notin J, i=2, \ldots, m$. Portanto

$$
v(a)=0, \quad v\left(a-c_{i}\right)=0, \quad i=2, \ldots, m
$$


isto é, $y=a$ é uma solução de (10) com $a \in F$. Isto completa a prova do caso $(i)$ $G=G^{\prime}$.

Caso (ii) $G \neq G^{\prime}$. Já que $F^{\prime}$ é de grau de transcendência 1 sobre $F, G^{\prime}$ tem que ser de grau 1 sobre $G$, por 1.12 . Seja $b$ um elemento arbitrário fixo de $G^{\prime}-G$, então todos os elementos de $G^{\prime}$ podem-se expressar de maneira única como

$$
d+n b, \quad d \in G, \quad n \in \mathbb{Q} .
$$

Seja $c \in F^{\prime}$ tal que $v(c)=b$. Então $c$ não pode estar em $F$, e $F(c)$ é uma extensão transcendental simples de $F$.

Denotemos por $K^{*}$ a união de $K, N$ (o diagrama de $M$ ), o conjunto de sentenças $\neg(a=c)$, onde $a$ varia sobre todas as constantes de $M$ (ver (3)), o conjunto adicional (13) definido como segue:

(13) Para cada $d \in G$, incluímos

$$
\neg(d=b) \wedge d \leq b \quad \text { ou } \quad \neg(d=b) \wedge b \leq d
$$

dependendo de qual destas sentenças seja verdadeira em $G^{\prime}$ (já que $G^{\prime}$ é uma extensão própria de $G$, uma das duas afirmações tem que ser verdadeira em cada caso); finalmente incluímos em $K^{*}$ a sentença $V(c, b) . M^{\prime}$ é um modelo de $K^{*}$ e qualquer outro modelo $M^{*}$ de $K^{*}$ compreende uma extensão própria $F^{*}$ de $F$ com grupo de valoração $G^{*}$, o qual é uma extensão própria de $G$. $F^{*}$ contém um elemento $c$ o qual é algebricamente independente de $F$, e de novo podemos supor que o corpo $F(c)$ está em $F^{\prime}$ e em $F^{*}$. Seja $\widehat{F}$ o fecho algébrico de $F(c)$ em $F^{*}$, e seja $\widehat{G}$ o grupo de valoração de $\widehat{F}, G \subseteq \widehat{G} \subseteq G^{*}$. $\widehat{G}$ contém uma constante $b$, a qual está relacionada com $c$ por $v(c)=b$, já que incluímos $V(c, b)$ em $K^{*}$.

Sejam $G_{0}^{\prime}, \widehat{G}_{0}$ os grupos de valoração de $F(c)$ em $G^{\prime}$ e em $\widehat{G}$ respectivamente. Em ambos os grupos $v(c)=b$. Então, por 1.12 (2) todos os elementos de cada grupo podem ser escritos da forma $d+n b$, onde $d \in G$ e $n$ é um inteiro, e as duas valorações 
coincidem por 1.12 e (13). Então 1.14 mostra que existe um isomorfismo $f$ entre $F^{\prime}$ e $\widehat{F}$ e um isomorfismo $h$ entre $G^{\prime}$ e $\widehat{G}$ os quais centralizam $F$ e $G$ tais que

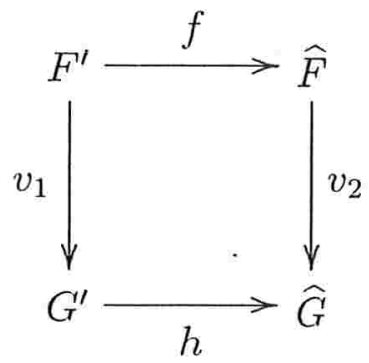

comuta. Isto prova que $\widehat{F}$ e $\widehat{G}$ constituem juntos um modelo $\widehat{M}$ no qual vale $\psi$, e concluímos então que $\psi$ vale em $M^{*}$, e portanto, é dedutível a partir de $K^{*}$. Assim, por (3) e (13) existe um número finito de elementos de $F, a_{1}, \ldots, a_{j}$ e um número finito de elementos de $G, d_{1}, \ldots, d_{l}, d_{l+1}, \ldots, d_{p}$ tais que a sentença

$$
\begin{aligned}
\neg\left(a_{1}=c\right) \wedge \cdots \wedge \neg\left(a_{j}=c\right) & \wedge \neg\left(d_{1}=b\right) \wedge d_{1} \leq b \wedge \cdots \wedge \neg\left(d_{l}=b\right) \wedge d_{l} \leq b \wedge \\
& \neg\left(d_{l+1}=b\right) \wedge b \leq d_{l+1} \wedge \cdots \wedge \neg\left(d_{p}=b\right) \wedge b \leq d_{p} \rightarrow \psi
\end{aligned}
$$

é dedutível de $K \cup N$. Nem $b$ nem $c$ estão em $M$ e para mostrar que $\psi$ vale em $M$ temos então que provar só que

$$
\begin{aligned}
\exists x \exists y\left(\neg\left(a_{1}=x\right) \wedge \cdots \wedge \neg\left(a_{j}=x\right)\right. & \wedge \neg\left(d_{1}=y\right) \wedge d_{1} \leq y \wedge \cdots \wedge \neg\left(d_{l}=y\right) \wedge d_{l} \leq y \wedge \\
& \left.\neg\left(d_{l+1}=y\right) \wedge y \leq d_{l+1} \cdots \wedge \neg\left(d_{p}=y\right) \wedge y \leq d_{p}\right)
\end{aligned}
$$

vale em $M$. Em linguagem algébrica, temos que mostrar que o sistema de equações dado nos seguintes (14) a (16) tem solução em $M$.

$$
\begin{array}{ll}
x \neq a_{i} & i=1, \ldots, j \\
y=v(x) & \\
y>d_{i} & i=1, \ldots, l, \\
y<d_{i} & i=l+1, \ldots p .
\end{array}
$$


Sabemos que o sistema possui uma solução em $M^{\prime}$, uma extensão de $M$. (16) é satisfeita por $y=b \in M^{\prime}$. Suponha que não pode ser satisfeita pelos elementos de $M$. e consideremos os seguintes casos: (a) nenhum dos conjuntos de (16) é vazio. Sejam

$$
\begin{aligned}
d^{\prime} & =\max \left\{d_{i}: 1 \leq i \leq l\right\} \\
d^{\prime \prime} & =\operatorname{mín}\left\{d_{i}: l+1 \leq i \leq p\right\}
\end{aligned}
$$

então $d^{\prime} \leq d^{\prime \prime}$ já que $d^{\prime}<b<d^{\prime \prime}$. Segue que

$$
y=a=\frac{1}{2}\left(d^{\prime}+d^{\prime \prime}\right) \in G
$$

é uma solução de (16) e $a \in G$ i.e. $a \in M$. Similarmente para um caso (b) quando um dos conjuntos é vazio. E para o caso (c) quando ambos os conjuntos são vazios nada temos a provar. Em todos os casos chegamos a uma contradição. Isto prova (16).

Tendo achado $y=y_{0}$ solução de (16) temos que determinar $x$ satisfazendo (14) e (15). Agora, já que $y_{0} \in G$, existe pelo menos um elemento $x_{0} \in F$, tal que $y_{0}=v\left(x_{0}\right)$. Escolhemos $j+1$ elementos diferentes de $F, e_{i}$, tais que $v\left(e_{i}\right)=0$ (e.g. as raízes de (12) para $q$ suficientemente grande). Então

$$
e_{1} x_{0}, e_{1} x_{0}, \ldots, e_{j+1} x_{0}
$$

são todos diferentes e $v\left(e_{i} x_{0}\right)=v\left(e_{i}\right)+v\left(x_{o}\right)=y_{0}$. Mas pelo menos um desses números, por exemplo $e_{1} x_{0}$, tem que ser diferente de todas as $a_{i}(i=1, \ldots, j)$. Então $x=e_{1} x_{0}$ satisfaz (14) e (15). Isto mostra que (14)-(16) tem solução em $M$ e completa a prova de 1.15 .

1.16 A noção de corpo valorado algebricamente fechado pode ser dada usando um sistema mais simples de relações. Sejam $E, S, P$ as relações que denotam a igualdade, 
a soma, e o produto. $\mathrm{E} R(x)$ uma relação unária (representa-se-à por $v(x) \geq 0$ ). Consideremos as seguintes sentenças

$$
\begin{aligned}
& \forall x y(R(x) \wedge E(x, y) \rightarrow R(y)) \\
& \forall x y z(R(x) \wedge R(y) \wedge S(x, y, z) \rightarrow R(z)) \\
& \forall x y z(R(x) \wedge R(y) \wedge S(x, z, y) \rightarrow R(z)) \\
& \forall x y z(R(x) \wedge R(y) \wedge P(x, y, z) \rightarrow R(z)) \\
& \forall x \exists y \exists z(R(y) \wedge R(z) \wedge P(x, y, z)) \\
& \forall x y \exists z(R(x) \wedge R(y) \rightarrow P(x, y, z) \vee P(x, z, y)) \\
& \exists x y \forall z w(R(x) \wedge R(y) \wedge \neg S(x, x, x) \wedge \neg S(y, y, y) \wedge \neg P(x, z, y) \wedge P(y, w, x)) \\
& \exists x(\neg R(x))
\end{aligned}
$$

Os axiomas anteriores determinam um subanel próprio $R$ do corpo dado, tal que todos os elementos do corpo podem ser representados como quociêntes de elementos do anel, e tal que para todo par de elementos do anel pelo menos um divide o outro. Sabe-se que (ver i.e. $[\mathrm{K}]$ ) um tal anel determina uma única valoração sobre o corpo. A última sentença indica que a valoração não é trivial.

Seja $K_{R}$ a união das sentenças dadas e os axiomas para a teoria dos corpos algebricamente fechados obtidos a partir de 1.2, 1.3, e 1.7. Então $K_{R}$ representa um conjunto de axiomas para o conceito de corpo algebricamente fechado com valoração não trivial. Uma prova análoga à do Teorema 1.15 mostra que $K_{R}$ é modelo completa (ver [R, (pg. 61)]).

\section{Eliminação de Quantificadores Para Corpos} Algebricamente Fechados com Valoração não Trivial

1.17 Seja $D$ um domínio. Uma relação linear de divisibilidade (1.d. relação) sobre $D$ 
é uma relação binária $L$ sobre $D$ satisfazendo:

$$
\begin{aligned}
& L(a, b) \wedge L(b, c) \rightarrow L(a, c) \\
& L(a, b) \vee L(b, a) \\
& L(a, b) \wedge L(a, c) \rightarrow L(a, b+c) \\
& (c \neq 0) \rightarrow(L(a, c) \equiv L(a c, b c)) \\
& \neg L(0,1)
\end{aligned}
$$

para todas $a, b, c \in D$. Uma l.d. relação sobre $D$ determina um anel de valoração $R_{L}$ no corpo de quociêntes $Q(D)$ de $D$. Este anel é dado por

$$
R_{L}=\{a / b: a, b \in D, b \neq 0, L(b, a)\} .
$$

E para a correspondente valoração $v_{L}$ sobre $Q(D)$ temos

$$
v_{L}(a) \leq v_{L}(b) \Longleftrightarrow L(a, b) \quad a, b \in D
$$

Claramente a transformação dada por $L \mapsto R_{L}$ é uma bijeção do conjunto de 1.d. relações sobre $D$ no conjunto de anéis de valoração de $Q(D)$, com inversa dada por

$$
R \mapsto L_{R}:=\left\{(a, b) \in D^{2}: v(a) \leq v(b)\right\},
$$

onde $v$ é a valoração sobre $Q(D)$ associada a $R$.

De novo, cada 1.d. relação $L$ sobre $D$ pode ser estendida de maneira única a uma 1.d. relação $L^{Q}$ sobre $Q(D)$ tal que

$$
(D, L) \subseteq\left(Q(D), L^{Q}\right) .
$$

Consideramos então um corpo valorado como um corpo com uma 1.d. relação sobre este. Seja $L_{d i v}$ a linguagem que contém as relações $E, S, P, L$. Analogamente a 1.16 obtemos uma axiomatização da teoria de corpos algebricamente fechados com valoração não trivial nesta linguagem. Denotamos esta teoria por $A C F_{\text {val }}$. 
Teorema 1.18. A teoria de corpos algebricamente fechados com valoração não trivial admite eliminação de quantificadores. (ver [M-McK-vdD, (pg. 83)])

Prova. Por 1.16 e $1.17 A C F_{v a l}$ é modelo completa. Cada corpo valorado possui uma única, salvo isomorfismo, extensão prima a um corpo valorado algebricamente fechado $\widetilde{F}$, com valoração não trivial. Se a valoração de $F$ é não trivial então a extensão prima é $\bar{F}$, o fecho algébrico junto com seu grupo de valoração. Se a valoração de $F$ é trivial, então primeiro estendemos a valoração a uma extensão pura transcendental de $K$, e depois aplicamos o primeiro caso. O Teorema 1.15 (modelo completude de corpos algebricamente fechados com valoração não trivial) junto com a existência de extensões primas para subestruturas implica que a teoria de corpos valorados algebricamente fechados admite eliminação de quantificadores (ver $[S]$ ). 



\section{CAPÍTULO 2}

\section{UM TEOREMA DE ELIMINAÇÃO DE QUANTIFICADORES PARA O DISCO UNITÁRIO DE $\overline{\mathbb{Q}}_{p}$}

Apresentamos neste capítulo um teorema de eliminação de quantificadores para uma expansão de $(I,+, \cdot, 0,1, P)$, onde $I$ denota o disco unitário de $\overline{\mathbb{Q}}_{p}$. E $\overline{\mathbb{Q}}_{p}$ denota o fecho algébrico de $\mathbb{Q}_{p}$.

J. Denef e L. van den Dries em [D-vd Dries, (1.1)] provam que $\mathbb{Z}_{p}$, o disco unitário de $\mathbb{Q}_{p}$, possui eliminação de quantificadores em uma linguagem obtida a partir de séries de potências convergentes em $\mathbb{Z}_{p}$, e que contém os predicados $P_{n}(x):=$ existe $y$ tal que $x=y^{n}$. A prova do teorema está baseada no fato de que $\mathbb{Z}_{p}$ é um anel noetheriano.

Claramente os predicados $P_{n}$ trivializam-se em $I$, e $I$ não é noetheriano. Assim propomos uma linguagem adequada que nos permite contornar essas dificuldades e provar um resultado análogo ao de Denef e van den Dries. 


\section{Notação e Resultados Preliminares}

Nesta seção fixamos a notação usada no que segue do capítulo, e apresentamos alguns resultados preliminares.

2.1 Seja $\overline{\mathbb{Q}}_{p}$ o fecho algébrico de $\mathbb{Q}_{p}$, e $I=\left\{x \in \overline{\mathbb{Q}}_{p}:|x| \leq 1\right\}$ o disco unitário de $\overline{\mathbb{Q}}_{p}$. Para $X=\left(X_{1}, \ldots, X_{m}\right)=X_{(m)}, \overline{\mathbb{Q}}_{p} \llbracket X \rrbracket$ denota o anel de séries de potências com coeficientes em $\overline{\mathbb{Q}}_{p}$, e $\overline{\mathbb{Q}}_{p}\{X\}$ o subanel de $\overline{\mathbb{Q}}_{p} \llbracket X \rrbracket$ que contém as séries $f=$ $\sum_{i \in \mathbb{N}^{m}} a_{i} X^{i}$ tais que $\left|a_{i}\right| \rightarrow 0$, quando $|i| \rightarrow \infty$, onde $|i|=i_{1}+\cdots+i_{m}$ para $i=\left(i_{1}, \ldots, i_{m}\right) \in \mathbb{N}^{m}$.

Pode-se ver que $\overline{\mathbb{Q}}_{p}$ não é completo para a norma $p$-ádica (ver $[G,(5.7 .4)]$ ).

2.2 Para $X=\left(X_{1}, \ldots, X_{n}\right), n \geq 0$, definimos

$$
\overline{\mathbb{Q}}_{p}\langle X\rangle:=\left\{f \in \overline{\mathbb{Q}}_{p} \llbracket X \rrbracket: \exists\left[K: \mathbb{Q}_{p}\right]<\infty, f \in K \llbracket X \rrbracket\right\},
$$

onde $\left[K: \mathbb{Q}_{p}\right]<\infty$ significa que $K$ é uma extensão finita de $\mathbb{Q}_{p}$, e para $n=0$ o anel correspondente é $\overline{\mathbb{Q}}_{p}$ : claramente $\overline{\mathbb{Q}}_{p}\langle X\rangle$ é um subanel de $\overline{\mathbb{Q}}_{p} \llbracket X \rrbracket$. Seja $I\{X\}$ o subanel de $\overline{\mathbb{Q}}_{p}\langle X\rangle \cap \overline{\mathbb{Q}}_{p}\{X\}$ que contém as séries $\sum a_{i} X^{i}$ tais que $a_{i} \in I$ para todo $i \in \mathbb{N}^{n}$. É fácil ver que para cada $f \in I\{X\}$, e cada $x \in I^{n}, f(x)$ é uma série convergente e $f(x) \in I$.

2.3 Definimos $D: I^{2} \rightarrow I$ por

$$
D\left(x_{1}, x_{2}\right)= \begin{cases}x_{1} / x_{2} & \text { se }\left|x_{1}\right| \leq\left|x_{2}\right|, \quad x_{2} \neq 0 \\ 0 & \text { caso contrario }\end{cases}
$$

2.4 Seja $L_{a n}^{D}$ a linguagem que contém um símbolo de operação $m$-ário $f$ para cada $f \in I\left\{X_{1}, \ldots, X_{m}\right\}, m \geq 0$, um símbolo de operação binário $D$, e um símbolo de relação binário $P$.

Nota. $P(x, y)$ representará $|y| \leq|x|$. 
2.5 $I$ é uma $L_{a n}^{D}$-estrutura de maneira natural. Observamos que para cada $\alpha \in I$ existe um símbolo de constante em $L_{a n}^{D}$ denotado por $\alpha$.

2.6 Omitindo o símbolo $D$, obtemos a linguagem $L_{a n}$. Claramente, para cada $L_{a n^{-}}$ termo $t\left(x_{1}, \ldots, x_{m}\right)$, existe uma série de potências $f \in I\left\{X_{1}, \ldots, X_{m}\right\}$ tal que $t(x)=$ $f(x)$ para toda $x \in I^{m}$.

Portanto qualquer $L_{a n}$-fórmula livre de quantificadores $\phi\left(X_{1}, \ldots, X_{m}\right)$ é equivalente a uma na qual todas as subfórmulas atômicas são da forma $f\left(X_{1}, \ldots, X_{m}\right)=0$ ou $P(f, g)$, com $f, g \in I\{X\}$.

Queremos provar neste capítulo que $I$ possui eliminação de quantificadores na linguagem $L_{a n}^{D}$.

Lema 2.7. Seja $\phi\left(X_{1}, \ldots, X_{M}\right)$ uma $L_{a n}^{D}$-fórmula livre de quantificadores na qual o símbolo $D$ ocorre $m$ vezes. Então existe uma $L_{a n}$-fórmula livre de quantificadores $\phi_{m}\left(X_{1}, \ldots, X_{M}, X_{M+1}, \ldots, X_{M+m}\right)$, tal que $I \models \phi \leftrightarrow \exists X_{M+1} \ldots X_{M+m} \phi_{m}$.

Prova. Se $m=0$, tomamos $\phi_{m}=\phi$. Se $m>0$ tomamos uma ocorrência de um termo $D\left(t_{1}, t_{2}\right)$ em $\phi$ tal que $D$ não ocorre nos termos $t_{1}, t_{2}$. Substituímos esta ocorrência de $D$ em $\phi$ por uma nova variável $X_{M+1}$; portanto obtemos uma fórmula $\bar{\phi}\left(X_{1}, \ldots, X_{M}, X_{M+1}\right)$, e seja $\phi_{1}\left(X_{1}, \ldots, X_{M}, X_{M+1}\right)$ a fórmula

$$
\bar{\phi} \wedge\left[\left(\left|t_{1}\right| \leq\left|t_{2}\right| \wedge t_{2} \neq 0 \wedge t_{1}=t_{2} X_{M+1}\right) \vee\left(\left(\left|t_{1}\right|>\left|t_{2}\right| \vee t_{2}=0\right) \wedge X_{M+1}=0\right)\right]
$$

(onde $\left|t_{1}\right|>\left|t_{2}\right|$ denota $\neg P\left(t_{2}, t_{1}\right)$ ). Então $I \models \phi \leftrightarrow \exists X_{M+1} \phi_{1}$. Agora $\phi_{1}$ contém só $m-1$ ocorrências do símbolo $D$; portanto se $m=1$ acabamos. Se $m>1$ repetimos o mesmo procedimento com $\phi_{1}$ no lugar de $\phi$. Depois de $m$ passos, obtemos $\phi_{m}$.

Nota. Observamos que se $S$ é o subconjunto de $I^{M}$ definido por $\phi$, e $S^{\prime}$ é o subconjunto de $I^{M+m}$ definido por $\phi_{m}$, então $S$ é a imagem injetiva de $S^{\prime}$ pela projeção canônica $\pi: I^{M+m} \rightarrow I^{M}$. 
2.8 A linguagem $L_{a n}$ contém símbolos de operação $\cdot,-$, e + , provenientes das séries $X_{1} \cdot X_{2},-X_{1}$, e $X_{1}+X_{2}$, respectivamente, e por 2.5 possui símbolos constantes 0,1 . Seja $L_{a, P}$ a sublinguagem de $L_{a n}$ contendo os símbolos $\{+,-, \cdot, 0,1, P\}$.

2.9 Dado um $L_{a n}^{D}$-termo $t\left(X_{1}, \ldots, X_{M+1}\right)$, dizemos que a variável $X_{M+1}$ ocorre polinomialmente em $t$, se este é da forma

$$
a_{d}\left(X_{1}, \ldots, X_{M}\right) X_{M+1}^{d}+a_{d-1}\left(X_{1}, \ldots, X_{M}\right) X_{M+1}^{d-1}+\cdots+a_{0}\left(X_{1}, \ldots, X_{M}\right),
$$

onde os $a_{i}\left(X_{1}, \ldots, X_{M}\right)$ são $L_{a n}^{D}$-termos com variáveis entre $X_{1}, \ldots, X_{M}$, e se $d=0$, então $t$ é $a_{0}\left(X_{1}, \ldots, X_{M}\right)$.

Seja $\phi\left(X_{1}, \ldots, X_{M}, X_{M+1}\right)$ uma $L_{a n}^{D}$-fórmula livre de quantificadores. Dizemos que a variável $X_{M+1}$ ocorre polinomialmente em $\phi$, se todas as subfórmulas atômicas de $\phi$ são da forma $t=0$ ou $P\left(t_{1}, t_{2}\right)$, com $X_{M+1}$ ocorrendo polinomialmente em $t, t_{1}, t_{2}$.

2.10 A $L_{a, P}$-estrutura $\left(\overline{\mathbb{Q}}_{p},+,-, \cdot, 0,1, P\right)$ possui eliminação de quantificadores. Para ver isto basta usar o Teorema de Eliminação de Quantificadores para corpos algebricamente fechados valorados (ver 1.18) e o fato de que

$$
P(x, y) \Longleftrightarrow|y| \leq|x| \Longleftrightarrow v(x) \leq v(y)
$$

é uma 1.d. relação sobre $\overline{\mathbb{Q}}_{p}$, onde $v$ denota a valoração p-ádica.

Claramente $\left(I,+,-, \cdot, 0,1,\left.P\right|_{I}\right)$ é uma $L_{a, P}$-subestrutura de $\left(\overline{\mathbb{Q}}_{p},+,-, \cdot, 0,1, P\right)$. A $L_{a, P}$-estrutura $\left(I,+,-, \cdot, 0,1,\left.P\right|_{I}\right)$ será denotada simplesmente por $(I,+,-, \cdot, 0,1, P)$.

Lema 2.11. Seja $\phi\left(X_{1}, \ldots, X_{M+1}\right)$ uma $L_{a n}^{D}$-fórmula livre de quantificadores na qual a variável $X_{M+1}$ ocorre polinomialmente. Então podemos obter uma $L_{a n}^{D}$-fórmula $\psi\left(X_{1}, \ldots, X_{M}\right)$ livre de quantificadores tal que

$$
I \models \exists X_{M+1} \phi \leftrightarrow \psi\left(X_{1}, \ldots, X_{M}\right) .
$$




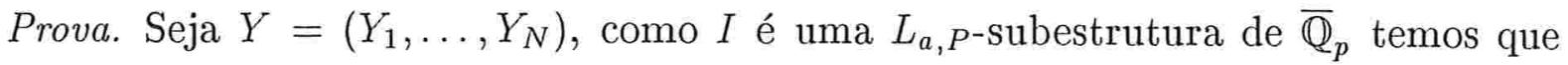
para cada $L_{a, P}$-fórmula livre de quantificadores $\phi^{\prime}\left(Y, X_{M+1}\right)$, e cada $y \in I^{N}$

$I \models \exists X_{M+1} \phi^{\prime}\left(Y, X_{M+1}\right)[y]$ se, e só se, $\overline{\mathbb{Q}}_{p} \models \exists X_{M+1}\left(\phi^{\prime}\left(Y, X_{M+1}\right) \wedge\left|X_{M+1}\right| \leq 1\right)[y]$.

De fato, suponhamos que

$$
I \models \exists X_{M+1} \phi^{\prime}\left(Y, X_{M+1}\right)[y],
$$

então existe $x \in I$ tal que

$$
I \models \phi^{\prime}\left(Y, X_{M+1}\right)[y, x],
$$

já que $I$ é $L_{a, P}$-subestrutura de $\overline{\mathbb{Q}}_{p}$, então

$$
\overline{\mathbb{Q}}_{p} \models \phi^{\prime}\left(Y, X_{M+1}\right)[y, x],
$$

como $x \in I$, então $|x| \leq 1$, e assim

$$
\overline{\mathbb{Q}}_{p} \models\left(\phi^{\prime}\left(Y, X_{M+1}\right) \wedge\left|X_{M+1}\right| \leq 1\right)[y, x],
$$

portanto

$$
\overline{\mathbb{Q}}_{p} \models \exists X_{M+1}\left(\phi^{\prime}\left(Y, X_{M+1}\right) \wedge\left|X_{M+1}\right| \leq 1\right)[y] .
$$

Reciprocamente, suponhamos que

$$
\overline{\mathbb{Q}}_{p} \models \exists X_{M+1}\left(\phi^{\prime}\left(Y, X_{M+1}\right) \wedge\left|X_{M+1}\right| \leq 1\right)[y],
$$

então existe $x \in \overline{\mathbb{Q}}_{p}$ tal que

$$
\overline{\mathbb{Q}}_{p} \models\left(\phi^{\prime}\left(Y, X_{M+1}\right) \wedge\left|X_{M+1}\right| \leq 1\right)[y, x] .
$$

Como $|x| \leq 1,(y, x) \in I^{N+1}$, e $\phi^{\prime}$ é livre de quantificadores, de novo usamos que $I$ é uma $L_{a, P}$-subestrutura de $\overline{\mathbb{Q}}_{p}$ para obter

$$
I \models\left(\phi^{\prime}\left(Y, X_{M+1}\right) \wedge\left|X_{M+1}\right| \leq 1\right)[y, x],
$$


portanto

$$
I \models \phi^{\prime}\left(Y, X_{M+1}\right)[y, x]
$$

e assim

$$
I \models \exists X_{M+1} \phi^{\prime}\left(Y, X_{M+1}\right)[y] .
$$

Agora, em cada subfórmula atômica de $\phi, t_{1}=0$ ou $P\left(t_{2}, t_{3}\right)$, os $t_{i}$ têm a forma $a_{d}(X) X_{M+1}^{d}+\cdots+a_{0}(X), \operatorname{com} X_{M+1}$ não ocorrendo nos $a_{j}(X) ;$ substituindo os $a_{j}(X)$ por novas variáveis $Y_{1}, Y_{2}, \ldots, Y_{N}$, obtemos $\phi^{\prime}\left(Y, X_{M+1}\right)$ livre de quantificadores na linguagem $L_{a, P}\left(Y=Y_{(N)}\right)$. Pelo Teorema de Eliminação de Quantificadores citado em 2.10, existe uma $L_{a, P}$-fórmula $\psi^{\prime}(Y)$ livre de quantificadores tal que para todo $y \in I^{N}$

$$
\overline{\mathbb{Q}}_{p} \models\left(\exists X_{M+1}\left(\phi^{\prime}\left(Y, X_{M+1}\right) \wedge\left|X_{M+1}\right| \leq 1\right) \leftrightarrow \psi^{\prime}(Y)\right)[y]
$$

portanto, para todo $y \in I^{N}$ temos

$$
I \models\left(\exists X_{M+1} \phi^{\prime}\left(Y, X_{M+1}\right) \leftrightarrow \psi^{\prime}(Y)\right)[y]
$$

Seja $\psi(X)$ a fórmula obtida ao substituir as $Y_{j}$ pelas $a_{j}(X)$ em $\psi^{\prime}(Y)$, então

$$
I \models \exists X_{M+1} \phi \leftrightarrow \psi(X) .
$$

Prova do Teorema Principal Assumindo o Lema Básico

Nesta seção assumiremos válido o seguinte Lema Básico (o qual será provado no final deste capítulo). 
Lema 2.12. (Básico) Seja $X=\left(X_{1}, \cdots, X_{M}\right), Y=\left(Y_{1}, \cdots, Y_{N}\right), N>0 . S e$ $\phi(X, Y)$ é uma $L_{a n}$-fórmula livre de quantificadores, então existe uma $L_{a n}^{D}$-fórmula livre de quantificadores $\psi\left(X, Z_{1}, \ldots, Z_{N-1}\right)$ tal que

(i) $I \models(\exists Y \phi(X, Y)) \leftrightarrow \exists Z_{1} \ldots Z_{N-1} \psi\left(X, Z_{1}, \ldots, Z_{N-1}\right)$.

(ii) em $\psi$ o símbolo de operação $D$ é aplicado só a termos que não envolvem as variáveis $Z_{1}, \ldots, Z_{N-1}$.

O lema básico pode ser estendido a $L_{a n}^{D}$-fórmulas livres de quantificadores $\phi$, nas quais $D$ é aplicado só a termos que não envolvem as variáveis $Y_{1}, \ldots, Y_{N}$ (ver $[B 2,(4.8)])$. De fato, seja $\phi$ uma dessas fórmulas, e sejam $t_{1}(X), \ldots, t_{k}(X)$ todos os subtermos de $\phi$ da forma

$$
t_{i}(X)=D\left(t_{i, 1}(X), t_{i, 2}(X)\right)
$$

onde $t_{i, 1}(X)$ e $t_{i, 2}(X)$ são $L_{a n}^{D}$-termos, $1 \leq i \leq k$. Sejam $W=\left(W_{1}, \ldots, W_{k}\right)$ novas variáveis, e seja $\tilde{\phi}(X, W, Y)$ a $L_{a n}^{D}$-fórmula obtida de $\phi(X, Y)$ ao se substituir todas as ocorrências de $t_{i}(X)$ em $\phi$ por $W_{i}$.

Podemos aplicar assim o Lema 2.12 a $\tilde{\phi}$, obtendo $\tilde{\psi}(X, W, Z), Z=\left(Z_{1}, \ldots, Z_{N-1}\right)$, satisfazendo $2.12(i)$ e $2.12(i i)$. Seja $\psi(X, Y)=\tilde{\psi}\left(X, t_{1}(X), \ldots, t_{k}(X), Z\right)$. Então

(i) $I \models(\exists Y \phi(X, Y)) \leftrightarrow \exists Z_{1} \ldots Z_{N-1} \psi\left(X, Z_{1}, \ldots, Z_{N-1}\right)$.

(ii) em $\psi$ o símbolo de operação $D$ é aplicado só a termos que não envolvem as variáveis $Z_{1}, \ldots, Z_{N-1}$.

Teorema 2.13. I admite eliminação de quantificadores na linguagem $L_{a n}^{D}$.

Prova. Aplicando a extensão do lema básico $N$ vezes, obtemos que, para cada $L_{a n}$-fórmula livre de quantificadores $\phi(X, Y)$, existe uma $L_{a n}^{D}$-fórmula livre de quantificadores $\psi(X)$ tal que $I \models \exists Y \phi(X, Y) \leftrightarrow \psi(X)$. 
Seja agora $\theta\left(X, Y_{1}\right)$ uma $L_{a n}^{D}$-fórmula livre de quantificadores. Temos que provar que existe uma $L_{a n}^{D}$-fórmula livre de quantificadores $\psi(X)$ tal que $I \models \exists Y_{1} \theta\left(X, Y_{1}\right) \leftrightarrow$ $\psi(X)$. Pelo Lema 2.7, podemos construir uma $L_{a n}$-fórmula livre de quantificadores $\phi\left(X, Y_{1}, Y_{2}, \ldots, Y_{N}\right)$, para algum $N>0$, tal que

$$
I \models \theta\left(X, Y_{1}\right) \leftrightarrow \exists Y_{2} \ldots Y_{N} \phi\left(X, Y_{1}, Y_{2}, \ldots, Y_{N}\right)
$$

Para esta $\phi$ existe uma $L_{a n}^{D}$-fórmula livre de quantificadores $\psi(X)$ tal que $I \models$ $\exists Y \phi(X, Y) \leftrightarrow \psi(X)$. Portanto $I \models \exists Y_{1} \theta\left(X, Y_{1}\right) \leftrightarrow \psi(X)$.

\section{Preliminares ao Lema BÁsico}

Apresentamos alguns resultados necessários à prova do Lema Básico. Usaremos a notação adotada na primeira seção deste capítulo. Assim, $I\{X\}$ é o subanel de $\overline{\mathbb{Q}}_{p}\{X\} \cap \overline{\mathbb{Q}}_{p}\langle X\rangle$ que contém as séries com coeficientes em $I$.

Para $f=\sum a_{i} X^{i} \in \overline{\mathbb{Q}}_{p}\{X\}, X=X_{(M)}$, definimos $\|f\|=\max \left\{\left|a_{i}\right|: i \in \mathbb{N}^{M}\right\}$.

Lema 2.14. Sejam $X=\left(X_{1}, \ldots, X_{M}\right), Y=\left(Y_{1}, \ldots, Y_{N}\right), N>0$. Se $f(X, Y) \in$ $I\{X, Y\}$, então $f$ pode ser escrita como $f=\sum a_{i}(X) Y^{i}$, onde i percorre $\mathbb{N}^{N}$ e $a_{i}=$ $a_{i}(X) \in I\{X\}$.

Prova. Por indução. Para $N=1$. Afirmamos que $f$ pode ser escrita como

$$
f\left(X, Y_{1}\right)=\sum_{j \in \mathbb{N}} a_{j}(X) Y_{1}^{j}, \quad \operatorname{com} a_{j}(X) \in I\{X\}
$$

Seja $a_{0}(X)=f(X, 0)$. Claramente $a_{0}(X) \in \overline{\mathbb{Q}}_{p}\langle X\rangle \cap \overline{\mathbb{Q}}_{p}\{X\}$, e os coeficientes de $a_{0}(X)$ estão em $I, \operatorname{assim} a_{0}(X) \in I\{X\}$.

Agora $f\left(X, Y_{1}\right)-a_{0}(X) \in I\left\{X, Y_{1}\right\}$ e $Y_{1}$ divide $f\left(X, Y_{1}\right)-a_{0}(X)$ em $\overline{\mathbb{Q}}_{p} \llbracket X, Y_{1} \rrbracket$. Portanto $f\left(X, Y_{1}\right)-a_{0}(X)=Y_{1} f_{1}\left(X, Y_{1}\right)$, para algum $f_{1} \in \overline{\mathbb{Q}}_{p} \llbracket X, Y_{1} \rrbracket$. Como $f-a_{0} \in$ 
$I\left\{X, Y_{1}\right\}$, então $f_{1}\left(X, Y_{1}\right) \in I\{X, Y\}$. De novo, $f_{1}(X, 0)=a_{1}(X) \in I\{X\}$. Por indução sobre $j \in \mathbb{N}$, usando o argumento anterior, segue que $a_{j}(X) \in I\{X\}$, para todo $j \in \mathbb{N}$.

Seja $N>1$. Pelo caso anterior, existem $a_{k}\left(X, Y_{1}, \ldots, Y_{N-1}\right) \in I\left\{X, Y_{1}, \ldots, Y_{N-1}\right\}$, $k \geq 0$, tais que

$$
f(X, Y)=\sum_{k \in \mathbb{N}} a_{k}\left(X, Y_{1}, \ldots, Y_{N-1}\right) Y_{N}^{k}
$$

pela hipótese de indução, para cada $k$ fixo, existem $a_{j, k}(X) \in I\{X\}, j \in \mathbb{N}^{N-1}$, tais que

$$
a_{k}\left(X, Y_{1}, \ldots, Y_{N-1}\right)=\sum_{j \in \mathbb{N}^{N-1}} a_{j, k}(X)\left(Y_{1}, \ldots, Y_{N-1}\right)^{j}
$$

portanto

$$
f(X, Y)=\sum_{j, k} a_{j, k}(X) Y^{(i, k)}
$$

2.15 Para um corpo $K$, tal que $\left[K: \mathbb{Q}_{p}\right]<\infty, I_{K}$ denota o disco unitário de $K$. Seja $I_{K}\{X\}$ o subanel de $K \llbracket X \rrbracket$ que contém as séries $f=\sum a_{i} X^{i}$, tais que, $a_{i} \in I_{K}$, e $\left|a_{i}\right| \rightarrow 0$ quando $|i| \rightarrow \infty$. Observamos que $I_{K}\{X\}$ é um subanel de $I\{X\}$.

Nota. No lema anterior observamos que se $f \in I\{X, Y\}$, então $f \in I_{K}\{X, Y\}$ para $\operatorname{algum}\left[K: \mathbb{Q}_{p}\right]<\infty$, e os $a_{i}(X) \in I_{K}\{X\}$.

2.16 Seja $A$ um anel, e $J$ um ideal de $A$ finitamente gerado. Então, o completado de $A$ com respeito a $J$ é noetheriano sempre que $A$ seja noetheriano (ver [Mat 1, $(23 . \mathrm{K})])$.

Lema 2.17. Para todo corpo $K$, com $\left[K: \mathbb{Q}_{p}\right]<\infty, I_{K}\left\{X_{1}, \ldots, X_{n}\right\}$ é noetheriano.

Prova. Já que $I_{K}$ é um anel de valoração discreta (ver $[\mathrm{G},(5.4 .2)]$ ), então $I_{K}$ é un domínio de ideais principais (ver[Mat 2,(11.1)]). Em particular $I_{K}$ é noetheriano, 
assim o anel de polinômios $A=I_{K}\left[X_{1}, \cdots, X_{n}\right]$ é noetheriano. Considere o ideal de $A$ dado por: $J=\sum_{i=1}^{n} p X_{i} A$. Então $I_{K}\{X\}$ é o completado de $A$ com respeito a $J$ (finitamente gerado). Portanto, por 2.16, $I_{K}\{X\}$ é noetheriano.

Lema 2.18. Sejam $X=\left(X_{1}, \ldots, X_{m}\right), Y=\left(Y_{1}, \ldots, Y_{n}\right), n>0$, e $f \in I\{X, Y\}$ escrita da forma $f=\sum a_{i} Y^{i}$ onde $i$ percorre $\mathbb{N}^{n}$ e $a_{i}=a_{i}(X) \in I\{X\}$. Então existe $d>0$ tal que para cada $i$, com $|i| \geq d$,

$$
a_{i}=\sum_{|j|<d} b_{i, j} a_{j}, \quad \operatorname{com} \quad b_{i, j} \in I\{X\}, \quad\left\|b_{i, j}\right\|<1 / p
$$

Além disso os $b_{i, j}$ podem ser escolhidos, para cada $j$ fixo com $|j|<d$, tais que $\left\|b_{i, j}\right\| \longrightarrow 0$ quando $|i| \longrightarrow \infty$.

Demonstração. Para cada $i \geq 0$ escrevemos $a_{i}=p^{\lambda(i)} \bar{a}_{i}, \operatorname{com} \lambda(i) \in \mathbb{N}, \bar{a}_{i} \in I\{X\}$ tal que $\lambda(i) \rightarrow \infty$ quando $|i| \rightarrow \infty$. Como $f \in I\{X, Y\}$ então existe um corpo $K$ tal que $\left[K: \mathbb{Q}_{p}\right]<\infty$, e se $I_{K}$ é o disco unitário de $K$, então $f \in I_{K}\{X, Y\}$, e $\bar{a}_{i}(X) \in I_{K}\{X\}$. Pelo lema anterior, $I_{K}\{X\}$ é noetheriano, e então o ideal gerado pelos $\bar{a}_{i}$, é realmente gerado por $\bar{a}_{j}$, com $|j|<d_{0}$, para algum inteiro $d_{0}>0$. Portanto para cada $i$ temos

$$
\bar{a}_{i}=\sum_{|j|<d_{0}} \bar{b}_{i, j} \bar{a}_{j}, \text { para alguns } \bar{b}_{i, j} \in I_{K}\{X\}
$$

Então $a_{i}=p^{\lambda(i)} \bar{a}_{i}=p^{\lambda(i)} \sum_{|j|<d_{0}} \bar{b}_{i, j} \bar{a}_{j}=p^{\lambda(i)} \sum_{|j|<d_{0}} \bar{b}_{i, j} a_{j} p^{-\lambda(j)}$. Logo, $a_{i}=$ $\sum_{|j|<d_{0}} p^{\lambda(i)-\lambda(j)} \bar{b}_{i, j} a_{j}$.

Escolhemos $d \geq d_{0}$ suficientemente grande para que $\lambda(i)-\lambda(j)>1$, para todo $i$ com $|i| \geq d$ e para todo $j$ com $|j|<d_{0}$.

Basta tomarmos 


$$
\begin{aligned}
& b_{i, j}=p^{\lambda(i)-\lambda(j)} \bar{b}_{i, j} \text { para }|i| \geq d, \quad|j|<d_{0} \\
& =0 \quad \text { para }|i| \geq d, \quad d_{0} \leq|j| \leq d
\end{aligned}
$$

Nota 2.19. Seja $f(X)=\sum a_{i}(X) Y^{i} \in I\{X\}$, como no lema anterior. Então para cada $x \in I^{M}$ e cada $|i| \geq d$, temos

$$
\begin{aligned}
\left|a_{i}(x)\right| & =\left|\sum_{|j|<d} b_{i, j}(x) a_{j}(x)\right| \\
& \leq \max \left\{\left|b_{i, j}(x) a_{j}(x)\right|:|j|<d\right\} \\
& \leq \max \left\{\left|a_{j}(x)\right|:|j|<d\right\}
\end{aligned}
$$

\section{Teorema De Divisão e Preparação de Weierstrass no}

\section{Caso não ARQUimediano}

2.20 Seja $F$ um corpo completo para uma ultramétrica, e $X=\left(X_{1}, \ldots, X_{n}\right)$. Denotamos com $F\{X\}$ o subanel de $F \llbracket X \rrbracket$ que contém as séries $f=\sum_{i \in \mathbb{N}^{n}} a_{i} X^{i}$, tais que, $\left|a_{i}\right| \rightarrow 0$, quando $|i| \rightarrow \infty$. Como antes, para cada $f \in F\{X\}$, definimos $\|f\|=\max \left\{\left|a_{i}\right|: i \in \mathbb{N}^{n}\right\}$.

O anel $\{f \in F\{X\}:\|f\| \leq 1\}$ é denotado por $F^{\prime}\{X\}$; o ideal $\left\{f \in F^{\prime}\{X\}:\|f\|<1\right\}$ é denotado por $F^{\prime \prime}\{X\}$, e $\bar{F}\{X\}$ representa $F^{\prime}\{X\} / F^{\prime \prime}\{X\}$. Em particular $\bar{F}=$ $F^{\prime} / F^{\prime \prime}$ denota o corpo residual de $F$. Já que $\bar{F}\{X\} \cong F[X]$ não tem divisores de zero, a norma sobre $F\{X\}$ é multiplicativa, isto é, $\|f g\|=\|f\|\|g\|$.

A sobrejeção canônica $F^{\prime}\{X\} \rightarrow \bar{F}\{X\}$ é denotada por $f \rightarrow \bar{f}$. Seja $X=$ $\left(X_{1}, \ldots, X_{n}\right)$ e $Y=Y_{1}$. Um elemento $f \in F\{X, Y\}$, com $\|f\|=1$, se chama 
regular de grau d em $Y$ se $\bar{f} \in \bar{F}\{X, Y\}$ é da forma

$$
\lambda Y^{d}+\sum_{i<d} c_{i}(X) Y^{i}
$$

onde $\lambda \in \bar{F}, \lambda \neq 0$ e $c_{0}, \ldots, c_{d-1} \in \bar{F}[X]$.

Teorema 2.21 (Divisão e Preparação de Weierstrass no Caso não Arquimediano). (ver [F-vd Put,(II.2.2)]) (1) Divisão. Seja $X=\left(X_{1}, \ldots, X_{n}\right), Y=Y_{1}$, e $f \in F\{X, Y\}$ regular de grau $d$ em $Y$. Então para toda $g \in F\{X, Y\}$ existem elementos únicos $q \in F\{X, Y\}$, e $r_{0}(X), \ldots, r_{d-1}(X) \in F\{X\}$ tais que

$$
g=f q+\sum_{i=0}^{d-1} r_{i}(X) Y^{i} .
$$

Além disso, se $r=\sum_{i=0}^{d-1} r_{i}(X) Y^{i}$,

$$
\|g\|=\max \{\|q\|,\|r\|\} .
$$

(2) Preparação. Se $f \in F\{X, Y\}$ é de norma 1 (i.e. $\|f\|=1$ ), então existe um $F$-automorfismo $\sigma$ de $F\{X, Y\}$, tal que $\sigma(f)$ é regular em $Y$.

Corolário 2.22. (ver [F-vd Put,(II.3.2)]) Seja $X=\left(X_{1}, \ldots, X_{n}\right), Y=Y_{1}, f \in$ $F\{X, Y\}$, f regular de grau d em $Y$. Então existe uma unidade u em $F\{X, Y\}$, e $r_{0}(X), \ldots, r_{d-1}(X) \in F\{X\}$, tais que

$$
f=u\left(Y^{d}+\sum_{i<d} r_{i}(X) Y^{i}\right) .
$$

2.23 Finalizamos esta seção observando que para toda extensão normal finita de $\mathbb{Q}_{p}$, $\left[K: \mathbb{Q}_{p}\right]<\infty, K$ é um corpo completo para a ultramétrica $p$-ádica (ver $[G,(5.3 .1)]$ ), e portanto os resultados anteriores valem para $K$. 
Prova do LEMA BÁsico

Os resultados apresentados nas seções anteriores deste capítulo permitem-nos provar agora o Lema 2.12 (Básico). Adaptamos, onde possível, a demonstração dada por J. Denef e L. van den Dries em [D-vd Dries,(1.5)].

2.24 Prova do Lema 2.12 (Básico). Seja $\varphi(X, Y)$ uma $L_{a n}$-fórmula livre de quantificadores. Então as subfórmulas atômicas de $\varphi$ são da forma $f(X, Y)=0$ e $P(f(X, Y), g(X, Y))$, com $f, g \in I\{X, Y\}$. Agora aplicamos o Lema 2.18 a todas as $f$ que ocorrem em $\varphi$ com um valor comum $d$ (justamente o maior). Fixamos o tal valor $d$, e consideramos cada $f(X, Y)$ tal que ou $f=0$ ou $P(f, g)$ ou $P(g, f)$ ocorrem como uma subfórmula de $\varphi$.

Adotamos a notação do Lema 2.18 para $f$, isto é, $f=\sum a_{i} Y^{i}$ onde $i$ percorre $\mathbb{N}^{n}$ e $a_{i}=a_{i}(X) \in I\{X\}$, e para cada $i, \operatorname{com}|i| \geq d$,

$$
a_{i}=\sum_{|j|<d} b_{i, j} a_{j}, \quad \text { com } b_{i, j} \in I\{X\}, \quad\left\|b_{i, j}\right\|<1 / p
$$

Além disso para cada $j$ fixo com $|j|<d,\left\|b_{i, j}\right\| \longrightarrow 0$ quando $|i| \longrightarrow \infty$. E seja $x=\left(x_{1}, \ldots, x_{M}\right)$ percorrendo $I^{M}$ no que segue.

Consideramos $Z_{f}(X)$ a $L_{a n}$-fórmula $\bigwedge_{|j|<d} a_{j}(X)=0$. Notemos que para cada $x$ temos

$$
I \models Z_{f}(x) \text { se, e só se, } f(x, y)=0 \quad \forall y \in I^{N} \text {, }
$$

Introduzimos para cada $j \in \mathbb{N}^{N}$, com $|j|<d$, conjuntos finitos $A(j)$ e $B(j)$ dados por

$$
A(j)=\{i:|i|<d, i<j\}, B(j)=\{i:|i|<d, i>j\}
$$


(Aqui $i<j$ refere-se à ordem lexicográfica em $\mathbb{N}^{N}$ ). Para cada $|j|<d$ seja $\mu_{f, j}$ a seguinte $L_{a n}$-fórmula

$$
a_{j}(X) \neq 0 \wedge\left(\bigwedge_{i \in A(j)}\left|a_{i}(X)\right| \leq\left|a_{j}(X)\right|\right) \wedge\left(\bigwedge_{i \in B(j)}\left|a_{i}(X)\right|<\left|a_{j}(X)\right|\right) .
$$

Portanto $\mu_{f, j}$ define em $I$ o conjunto de $x \in I^{M}$ para o qual $j$ é o elemento lexicograficamente maior de $\mathbb{N}^{N} \operatorname{com}\left|a_{j}(x)\right|=\max \left\{\left|a_{i}(x)\right|: i \in \mathbb{N}^{N}\right\}$, já que $d$ é como no Lema 2.18 (ver Nota 2.19). Notemos que os conjuntos definidos pelas fórmulas $Z_{f}(X)$ e $\mu_{f, j}(X),|j|<d$, são disjuntos e cobrem $I^{M}$.

Centramos agora nossa atenção sobre um índice fixo $j \in \mathbb{N}^{N}$ com $|j|<d$. A idéia agora é que se $I \models \mu_{f, j}(X)$, então, depois de uma transformação das $Y$-variáveis as quais não dependem de $X$, o Teorema de Preparação de Weierstrass no Caso não Arquimediano pode-se aplicar a $a_{j}^{-1}(X) f(X, Y)$. Para realizar esta idéia precisamos de uma tupla $V=\left(V_{i}\right)_{i \in A(j) \cup B(j)}$ de variáveis auxiliares (suprimimos a dependência de $V$ sobre $j$ para não sobrecarregar a notação).

Separamos $f$ da seguinte maneira

$$
\begin{aligned}
f= & \left(\sum_{i \in A(j)} a_{i} Y^{i}\right)+a_{j} Y^{j}+\left(\sum_{i \in B(j)} a_{i} Y^{i}\right) \\
& +\sum_{|i| \geq d}\left\{\left(\sum_{k \in A(j)} b_{i, k} a_{k}\right)+b_{i, j} a_{j}+\left(\sum_{k \in B(j)} b_{i, k} a_{k}\right)\right\} Y^{i},
\end{aligned}
$$

onde os $b$ são como no Lema 2.18. Dividimos nesta fórmula todos os $a_{i}$ pelos $a_{j}$, substituindo todos os cocientes resultantes $a_{i} / a_{j}$ por $V_{i}$ ou por $p V_{i}$ de acordo com $i \in A(j)$ ou $i \in B(j)$ resultando na série $\tilde{f} \in I \llbracket X, V, Y \rrbracket$ definida por

$$
\begin{aligned}
\tilde{f}= & \left(\sum_{i \in A(j)} V_{i} Y^{i}\right)+Y^{j}+\left(\sum_{i \in B(j)} p V_{i} Y^{i}\right) \\
& +\sum_{|i| \geq d}\left\{\left(\sum_{k \in A(j)} b_{i, k} V_{k}\right)+b_{i, j}+\left(\sum_{k \in B(j)} b_{i, k} p V_{k}\right)\right\} Y^{i}
\end{aligned}
$$


Já que $\left\|b_{i k}\right\| \longrightarrow 0$ quando $|i| \longrightarrow \infty$, temos $\tilde{f} \in I\{X, V, Y\}$. Claramente $\tilde{f}$ está construída tal que

$$
\text { Se } \quad I \models \mu_{f, j}(x), \quad \text { então } \quad f(x, Y)=a_{j}(x) \widetilde{f}(x, v(x), Y) \text {, }
$$

onde $v(x)=\left(v_{i}(x)\right)_{i \in A(j) \cup B(j)}$, com

$$
\begin{aligned}
v_{i}(x) & =D\left(a_{i}(x), a_{j}(x)\right) & \text { se } & i \in A(j) \\
& =D\left(a_{i}(x), p a_{j}(x)\right) & \text { se } & i \in B(j)
\end{aligned}
$$

(também definimos $v(x)$ desta maneira se $I \models \neg \mu_{f, j}(x)$ ).

Sejam $Z_{1}, \cdots, Z_{N}$, novas variáveis, $Z=\left(Z_{1}, \cdots, Z_{N}\right)$, e seja

$$
T: I\{X, V, Y\} \longrightarrow I\{X, V, Z\}
$$

o isomorfismo de $I\{X, V\}$-álgebras dado por

$$
T\left(Y_{\lambda}\right)=Z_{\lambda}+\left(Z_{N}\right)^{d^{N-\lambda}} \text { para } 1 \leq \lambda<N, \quad T\left(Y_{N}\right)=Z_{N}
$$

Sob esta transformação $\tilde{f}$ se torna $T(\widetilde{f})$, e já que $\left\|b_{i, j}\right\|<1 / p$, temos:

$$
T(\tilde{f}) \equiv\left(\sum_{i \in A(j)} V_{i}\left(T Y_{1}\right)^{i_{1}} \cdots\left(T Y_{N}\right)^{i_{N}}\right)+\left(T Y_{1}\right)^{j_{1}} \cdots\left(T Y_{N}\right)^{j_{N}} \bmod p
$$

Seja $E=j_{N}+j_{N-1} d+\cdots+j_{1} d^{N-1}$, então

$$
T(\tilde{f}) \equiv C_{0}+C_{1} Z_{N}+\cdots+C_{E-1} Z_{N}^{E-1}+Z_{N}^{E} \bmod p
$$

onde $C_{i} \in I\left[V, Z_{1}, \cdots, Z_{N-1}\right]$, pois $j$ é lexicograficamente maior que todo $i \in A(j)$. Assim $T(\tilde{f})$ é regular de grau $E$ em $Z_{N}$. Podemos aplicar agora o Teorema de 
Preparação de Weierstrass no Caso não Arquimediano, dado em 2.21, a $T(\widetilde{f})$; e pelo Colorário 2.22, temos

$$
T(\tilde{f})=U\left(Z_{N}^{E}+A_{1} Z_{N}^{E-1}+\cdots+A_{E}\right)
$$

para alguma unidade $U$ de $I\{X, V, Z\}$ e certas $A_{1}, \ldots, A_{E} \in I\left\{X, V, Z_{1}, \ldots, Z_{N-1}\right\}$. Observemos o que isto significa para a série original $f$ considerada como uma função sobre $I^{M+N}$. Associamos a cada $y \in I^{N}$ um ponto $z(y)=\left(z_{1}(y), \ldots, z_{n}(y)\right) \in I^{N}$ por:

$$
\begin{aligned}
& y_{\lambda}=z_{\lambda}(y)+z_{N}(y)^{d^{N-\lambda}} \quad \text { para } \quad 1 \leq \lambda<N \\
& y_{N}=z_{N}(y) .
\end{aligned}
$$

Notemos que a transformação $I^{N} \rightarrow I^{N}: y \mapsto z(y)$ é uma bijeção. Para simplificar, escreveremos $\bar{A}_{\mu}$ para $A_{\mu}\left(x, v(x), z_{1}(y), \cdots, z_{N-1}(y)\right), 1 \leq \mu \leq E$.

Combinando (1) e (2), obtemos: Se $I \models \mu_{f, j}$, então $\forall y \in I^{N}$,

$$
f(x, y)=a_{j}(x) U(x, v(x), y(z))\left(z_{N}(y)^{E}+\bar{A}_{1} z_{N}(y)^{E-1}+\cdots+\bar{A}_{E}\right) .
$$

Portanto, se $I \models \mu_{f, j}(x)$, então $\forall y \in I^{N}$

$$
f(x, y)=0 \Longleftrightarrow z_{N}(y)^{E}+\bar{A}_{1} z_{N}(y)^{E-1}+\cdots+\bar{A}_{E}=0 .
$$

Agora consideramos dois casos

(a) $f$ aparece em $\phi$ como uma subfórmula $f(X, Y)=0$.

(b) $f$ aparece em $\phi$ como uma subfórmula $P(f, g)$ ou $P(g, f)$.

No caso (a) notemos que $f(X, Y)=0$ é equivalente a

$$
Z_{f}(X) \vee \bigvee_{|j|<d}\left(\mu_{f, j}(X) \wedge f(X, Y)=0\right)
$$


Além disso, se $|j|<d$, devido a (4) existe uma $L_{a n}^{D}$-fórmula $\psi_{0, j}(X, Z)$ livre de quantificadores na qual $D$ é aplicado só a termos que não envolvem $Z_{1}, \cdots, Z_{N}$, e $Z_{N}$ ocorre só polinomialmente, tal que para todo $(x, y) \in I^{M+N}$,

$$
I \models\left(\mu_{f, j}(x) \wedge f(x, y)=0\right) \leftrightarrow\left(\mu_{f, j}(x) \wedge \psi_{0, j}(x, z(y)) .\right.
$$

Aqui usamos que a função $\left(x, z_{1}, \ldots, z_{N-1}\right) \mapsto A_{\mu}\left(x, v(x), z_{1}, \cdots, z_{N-1}\right)$, definida em $I^{M+N-1}$, é dada por um termo $\tau_{\mu}\left(X, Z_{1}, \cdots, Z_{N-1}\right)$ de $L_{a n}^{D}$ no qual $D$ é aplicado só a subtermos que não envolvem $Z_{1}, \cdots, Z_{N-1}$. Seja $\psi_{0}(X, Z)$ a fórmula

$$
Z_{f} \vee \bigvee_{|j|<d}\left(\mu_{f, j}(X) \wedge \psi_{0, j}(X, Z)\right)
$$

Os argumentos anteriores mostram

$$
I \models f(X, Y)=0 \leftrightarrow \psi_{0}(X, Z(Y))
$$

onde $Z(Y)$ consiste de $\left(Y_{1}-Y_{N}^{d^{N-1}}, \cdots, Y_{N-1}-Y_{N}^{d}, Y_{N}\right)$.

Agora tratamos o caso (b). Consideremos a fórmula $|f| \leq|g|$ onde $f, g \in I\{X, Y\}$. Como indicamos no início da prova, usaremos a notação dada pelo Lema 2.18. Isto é,

$$
f=\sum a_{i}(X) Y^{i} \text { e } g=\sum b_{i}(X) Y^{i}
$$

onde os $a_{i}(X)$ e os $b_{i}(X)$ satisfazem o Lema 2.18 para um valor comum $d$.

Além de $Z_{f}$ e $\mu_{f, j}$, consideramos as fórmulas: $Z_{g}(X)$ dada por $\Lambda_{|j|<d} b_{j}(X)=0 \mathrm{e}$ $\mu_{g, j}$ definida por

$$
b_{j}(X) \neq 0 \wedge\left(\bigwedge_{i \in A(j)}\left|b_{i}(X)\right| \leq\left|b_{j}(X)\right|\right) \wedge\left(\bigwedge_{i \in B(j)}\left|b_{i}(X)\right|<\left|b_{j}(X)\right|\right) .
$$

Já que

$$
|f| \leq|g| \text { se, e só se } f=0 \vee(g=0 \wedge f=0) \vee((f \neq 0 \wedge g \neq 0) \wedge|f| \leq|g|) \text {, }
$$


podemos supor $f(X, Y) \neq 0$, e $g(X, Y) \neq 0$ (caso contrário usamos o caso (a)). Como $f \neq 0 \wedge g \neq 0$ é equivalente a $f g \neq 0$, assumiremos $f g(X, Y)=f(X, Y) g(X, Y) \neq 0$.

Observe que para todo $(x, y) \in I^{M+N}, f(x, y) \neq 0$ implica $\mu_{f, j}(x)$ para algum $j$ com $|j|<d$ (senão $Z_{f}(x)$ implica $f(x, y)=0$ ). Igualmente $g(x, y) \neq 0$ implica $\mu_{g, k}(x)$ para algum $k$ com $|k|<d$. Temos assim que

$$
I \models f g(X, Y) \neq 0 \leftrightarrow f g(X, Y) \neq 0 \wedge\left(\bigvee_{|j|,|k|<d} \mu_{f, j}(X) \wedge \mu_{g, k}(X)\right) .
$$

Portanto

$$
\begin{aligned}
I \models f g(X, Y) & \neq 0 \wedge|f(X, Y)| \leq|g(X, Y)| \leftrightarrow \\
f g(X, Y) \neq 0 & \wedge\left(\bigvee_{|j|,|k|<d} \mu_{f, j}(X) \wedge \mu_{g, k}(X) \wedge|f(X, Y)| \leq|g(X, Y)|\right) .
\end{aligned}
$$

Agora, se $I \models \mu_{f, j}(x) \wedge \mu_{g, k}(x)$ então $\forall y \in I^{N}$

$$
f(x, y)=a_{j}(x) U(x, v(x), z(y))\left(z_{N}(y)^{E}+\bar{A}_{1} z_{N}(y)^{E-1}+\cdots+\bar{A}_{E}\right)
$$

e

$$
g(x, y)=b_{k}(x) U^{\prime}(x, v(x), z(y))\left(z_{N}(y)^{E^{\prime}}+\bar{B}_{1} z_{N}(y)^{E^{\prime}-1}+\cdots+\bar{B}_{E^{\prime}}\right)
$$

onde $E^{\prime}=k_{N}+k_{N-1} d+\cdots+k_{1} d^{N-1}$, e $U(x, v, z), U^{\prime}(x, v, z)$ são unidades de $I\{X, V, Z\}\left(Z=Z_{1}, \ldots, Z_{N}\right)$, e os $\bar{A}_{\alpha}=A_{\alpha}\left(x, v(x), z_{1}(y), \ldots, z_{N-1}(y)\right), \bar{B}_{\alpha}=$ $B_{\alpha}\left(x, v(x), z_{1}(y), \ldots, z_{N-1}(y)\right.$, são como em (2).

Assim, para cada tupla $(x, v, z), U(x, v, z)$ e $U^{\prime}(x, v, z)$ são unidades de $I$, portanto $|U(x, v, z)|=\left|U^{\prime}(x, v, z)\right|=1$. Assim se $I \models \mu_{f, j}(x) \wedge \mu_{g, k}(x)$ então $\forall y \in I^{N}$,

$$
\begin{aligned}
|f(x, y)| & =\left|a_{j}(x)\right||U(x, v(x), z(y))|\left|\left(z_{N}(y)^{E}+\bar{A}_{1} z_{N}(y)^{E-1}+\cdots+\bar{A}_{E}\right)\right| \\
& =\left|a_{j}(x)\right|\left|\left(z_{N}(y)^{E}+\bar{A}_{1} z_{N}(y)^{E-1}+\cdots+\bar{A}_{E}\right)\right|
\end{aligned}
$$


$\mathrm{e}$

$$
\begin{aligned}
|g(x, y)| & =\left|b_{k}(x)\right|\left|U^{\prime}(x, v(x), z(y))\right|\left|\left(z_{N}(y)^{E^{\prime}}+\bar{B}_{1} z_{N}(y)^{E^{\prime}-1}+\cdots+\bar{B}_{E^{\prime}}\right)\right| \\
& =\left|b_{k}(x)\right|\left|\left(z_{N}(y)^{E^{\prime}}+\bar{B}_{1} z_{N}(y)^{E^{\prime}-1}+\cdots+\bar{B}_{E^{\prime}}\right)\right| .
\end{aligned}
$$

Existe então uma $L_{a n}^{D}$-fórmula livre de quantificadores $\psi_{1}^{\prime}\left(X, Z_{1}, \ldots, Z_{N}\right)$ na qual $D$ é aplicado unicamente a termos que não envolvem $Z_{1}, \ldots, Z_{N}$, e $Z_{N}$ ocorre polinomialmente tal que

$$
I \models f g(X, Y) \neq 0 \wedge|f(X, Y)| \leq|g(X, Y)| \leftrightarrow f g(X, Y) \neq 0 \wedge \psi_{1}^{\prime}(X, Z) .
$$

Finalmente (usando o caso (a)) temos que existe uma $L_{a n}$-fórmula livre de quantificadores $\psi_{1}\left(X, Z_{1}, \ldots, Z_{N}\right)$ na qual $D$ é aplicado unicamente a termos que não envolvem $Z_{1}, \ldots, Z_{N-1}$, e $Z_{N}$ ocorre polinomialmente tal que

$$
I \models|f(X, Y)| \leq|g(X, Y)| \leftrightarrow \psi_{1}(X, Z) .
$$

De (5) e (6) obtemos uma $L_{a n}^{D}$-fórmula livre de quantificadores $\phi^{d}(X, Z)$ na qual $D$ é aplicado unicamente a termos que não envolvem $Z_{1}, \ldots, Z_{N-1}$, e $Z_{N}$ ocorre só polinomialmente, tal que

$$
I \models \phi(X, Y) \leftrightarrow \phi^{d}(X, Z(Y))
$$

Dado que $y \mapsto z(y)$ é uma permutação de $I^{N}$, isto implica que

$$
I \models \exists Y \phi(X, Y) \leftrightarrow \exists Z \phi^{d}(X, Z)
$$

Já que em $\phi^{d}(X, Z)$ a variável $Z_{N}$ ocorre só polinomialmente podemos aplicar o Lema 2.11 a $\exists Z_{N} \phi^{d}(X, Z)$. Obtemos uma $L_{a n}^{D}$-fórmula livre de quantificadores $\psi\left(X, Z_{1}, \cdots, Z_{N-1}\right)$ tal que 
(9)

$$
I \models \exists Z_{N} \phi^{d}(X, Z) \longleftrightarrow \psi\left(X, Z_{1}, \cdots, Z_{N-1}\right) .
$$

Combinando (8) e (9) obtemos a equivalência desejada

$$
I \models \exists Y \phi(X, Y) \longleftrightarrow \exists Z_{1} \cdots \exists Z_{N-1} \psi\left(X, Z_{1}, \cdots, Z_{N-1}\right)
$$




\section{CAPÍTULO 3}

\section{UM TEOREMA DE ELIMINAÇÃO DE QUANTIFICADORES PARA O DISCO UNITÁRIO DE $\mathbb{C}_{p}$}

Neste capítulo provaremos que o disco unitário de $\mathbb{C}_{p}$ possui eliminação de quantificadores na linguagem $L_{a n}^{D}$ introduzida no Capítulo 2.

As provas dos principais resultados são análogas às apresentadas no capítulo anterior, e serão omitidas. 


\section{Notação e Resultados Preliminares}

Nesta seção fixamos a notação usada no que segue do capítulo, e apresentamos alguns resultados preliminares.

3.1 Seja $\mathbb{C}_{p}$ o completamento de $\overline{\mathbb{Q}}_{p}$ com respeito à norma $p$-ádica, e $\mathcal{D}=\{x \in$ $\left.\mathbb{C}_{p}:|x| \leq 1\right\}$ o disco unitário de $\mathbb{C}_{p}$. Notemos que $\mathbb{C}_{p}$ é algebricamente fechado (ver $[\mathrm{G},(5.7 .8)])$.

Para $X=\left(X_{1}, \ldots, X_{n}\right)$, lembramos que $I\{X\}$ é o subanel de $\overline{\mathbb{Q}}_{p} \llbracket X \rrbracket$ que contém as séries $f=\sum_{i \in \mathbb{N}^{n}} a_{i} X^{i}$ com coeficientes em $I_{K}$, o disco unitário de $K$, uma extensão normal finita de $\mathbb{Q}_{p}\left(\left[K: \mathbb{Q}_{p}\right]<\infty\right)$, tais que $\left|a_{i}\right| \rightarrow 0$, quando $|i| \rightarrow \infty$.

Observemos que para todo $x \in \mathcal{D}^{n}$ e $f \in I\{X\}, f(x)$ é uma série convergente, e $f(x) \in \mathcal{D}$.

3.2 Definimos $D: \mathcal{D}^{2} \rightarrow \mathcal{D}$ por

$$
D\left(x_{1}, x_{2}\right)= \begin{cases}x_{1} / x_{2} & \text { se }\left|x_{1}\right| \leq\left|x_{2}\right|, \quad x_{2} \neq 0 \\ 0 & \text { caso contrario }\end{cases}
$$

Lembramos que $L_{a n}^{D}$ é a linguagem que contém um símbolo de operação $m$-ário $f$ para cada $f \in I\left\{X_{1}, \ldots, X_{m}\right\}, m \geq 0$, um símbolo de operação binário $D$, e um símbolo de relação binário $P$ (onde $P(x, y)$ é também denotado por $|y| \leq|x|$ ).

Claramente $\mathcal{D}$ é uma $L_{a n}^{D}$-estrutura com as interpretações naturais. Se $X=$ $\left(X_{1}, \ldots, X_{n}\right)$, observemos que para toda $f \in I\{X\}$, e todo $x \in I^{n}, f(x) \in I$. Portanto $\left(I,(f)_{f \in I\{X\}}, D, P\right)$ é uma $L_{a n}^{D}$-subestrutura de $\left(\mathcal{D},(f)_{f \in I\{X\}}, D, P\right)$.

Por 2.10, omitindo o símbolo $D$, obtemos a linguagem $L_{a n}$. Lembramos que qualquer $L_{a n}$-fórmula livre de quantificadores $\phi\left(X_{1}, \ldots, X_{m}\right)$ é equivalente a uma fórmula na qual todas as subfórmulas atômicas são da forma $f\left(X_{1}, \ldots, X_{m}\right)=0$ ou $P(f, g)$, $\operatorname{com} f, g \in I\{X\}$.

Neste capítulo queremos provar que $\mathcal{D}$ possui eliminação de quantificadores na linguagem $L_{a n}^{D}$. 
Substituindo $I$ por $\mathcal{D}$ no Lema 2.7 obtemos o seguinte

Lema 3.3. Seja $\phi\left(X_{1}, \ldots, X_{M}\right)$ uma $L_{a n}^{D}$-fórmula livre de quantificadores na qual o símbolo $D$ ocorre $m$ vezes. Então existe uma $L_{a n}$-fórmula livre de quantificadores $\phi_{m}\left(X_{1}, \ldots, X_{M}, X_{M+1}, \ldots, X_{M+m}\right)$, tal que $\mathcal{D} \models \phi \leftrightarrow \exists X_{M+1} \ldots X_{M+m} \phi_{m}$.

Como antes $L_{a, P}$ é a sublinguagem de $L_{a n}$ contendo os símbolos $\{+,-, \cdot, 0,1, P\}$. $3.4\left(\mathbb{C}_{p},+,-, \cdot, 0,1, P\right)$ possui eliminação de quantificadores na linguagem $L_{a, P}$. De novo, basta usar o teorema de eliminação de quantificadores para corpos algebricamente fechados valorados (ver Teorema 1.18) e o fato de que

$$
P(x, y) \Longleftrightarrow|y| \leq|x| \Longleftrightarrow v(x) \leq v(y)
$$

é uma l.d. relação sobe $\mathbb{C}_{p}$, onde $v$ denota a valoração p-ádica.

Observamos que $\left(\mathcal{D},+,-, \cdot, 0,1,\left.P\right|_{\mathcal{D}}\right)$ é uma $L_{a, P}$-subestrutura de $\left(\mathbb{C}_{p},+,-, \cdot, 0,1, P\right)$. A estrutura $\left(\mathcal{D},+,-, \cdot, 0,1,\left.P\right|_{\mathcal{D}}\right)$ será notada simplesmente por $(\mathcal{D},+,-, \cdot, 0,1, P)$.

Substituindo $I$ por $\mathcal{D}$, e $\overline{\mathbb{Q}}_{p}$ por $\mathbb{C}_{p}$, na prova do Lema 2.11 , obtemos o seguinte

Lema 3.5. Seja $\phi\left(X_{1}, \ldots, X_{M+1}\right)$ uma $L_{a n}^{D}$-fórmula livre de quantificadores na qual a variável $X_{M+1}$ ocorre polinomialmente. Então podemos obter uma $L_{a n}^{D}$-fórmula $\psi\left(X_{1}, \ldots, X_{M}\right)$ livre de quantificadores tal que

$$
\mathcal{D} \models \exists X_{M+1} \phi \leftrightarrow \psi\left(X_{1}, \ldots, X_{M}\right)
$$

\section{Prova do Teorema Principal}

Observamos que o Teorema de Divisão e Preparação de Weierstrass dado em 2.21 vale para $\mathbb{C}_{p}$.

Uma prova idêntica à dada em 2.24 (com as substituições óbvias), demonstra o seguinte 
Lema 3.6. (Básico) Seja $X=\left(X_{1}, \cdots, X_{M}\right), Y=\left(Y_{1}, \cdots, Y_{N}\right), N>0$. Se $\phi(X, Y)$ é uma $L_{a n}$-fórmula livre de quantificadores, então existe uma $L_{a n}^{D}$-fórmula livre de quantificadores $\psi\left(X, Z_{1}, \ldots, Z_{N-1}\right)$ tal que

(i) $\mathcal{D} \models(\exists Y \phi(X, Y)) \longleftrightarrow \exists Z_{1} \ldots Z_{N-1} \psi\left(X, Z_{1}, \ldots, Z_{N-1}\right)$.

(ii) em $\psi$ o símbolo de operação $D$ é aplicado só a termos que não envolvem as variáveis $Z_{1}, \ldots, Z_{N-1}$.

Assim, repetindo a prova dada em 2.13 obtemos o seguinte

Teorema 3.7. $\mathcal{D}$ admite eliminação de quantificadores na linguagem $L_{a n}^{D}$. 
CAPÍTULO 4

CONJUNTOS SEMI-ALGÉBRICOS EM $\mathbb{C}_{p}$ E $\overline{\mathbb{Q}}_{p}$,
SEMI-ANALÍTICOS E SUBANALÍTICOS DE $\mathbb{C}_{p}$

Usando o predicado $P(x, y):|y| \leq|x|$ introduzimos as noções de subconjunto semianalítico de $\mathbb{C}_{p}^{M}$ (resp. $\overline{\mathbb{Q}}_{p}^{M}$ ), e provamos alguns fatos básicos sobre eles. Baseados no trabalho de Serre [Se], formulamos as noções de função analítica p-ádica complexa, e variedade analítica p-ádica complexa. Utilizando as noções anteriores definimos o conceito de subconjunto subanalítico de uma variedade analítica $p$-ádica complexa. Finalmente provamos um teorema de existência de cotas para conjuntos subanalíticos de $\mathbb{C}_{p}$. 


\section{Conjuntos Semi-Algébricos de $\mathbb{C}_{p}$ E $\overline{\mathbb{Q}}_{p}$}

4.1 Um subconjunto $A \subseteq \mathbb{C}_{p}^{M}$ é chamado semi-algébrico se é um elemento da álgebra de Boole gerada pelos conjuntos da forma

$$
\left\{x \in \mathbb{C}_{p}^{M}: f(x)=0,|g(x)| \leq|h(x)|\right\},
$$

onde $f, g, h \in \mathbb{C}_{p}\left[X_{1}, \ldots, X_{M}\right]$.

Lembramos que $L_{a, P}$ é a linguagem que contém os símbolos $+, \cdot, 0,1, P$ (onde $P(X, Y)$ denota $|X| \leq|Y|)$. Seja $L_{a, P}^{\mathbb{C}_{p}}$ a linguagem obtida ao agregar um símbolo de constante para cada elemento de $\mathbb{C}_{p}$.

4.2 Pelo Teorema 1.18 (Eliminação de Quantificadores para Corpos Valorados Algebricamente Fechados), $\mathbb{C}_{p}$ possui eliminação de quantificadores na linguagem $L_{a, P}^{\mathbb{C}_{p}}$.

Claramente um subconjunto $A \subseteq \mathbb{C}_{p}^{M}$ é semi-algébrico se, e só se, $A$ é definido por uma $L_{a, P}^{\mathbb{C}_{p}}$-fórmula livre de quantificadores.

Lema 4.3. Seja $A$ um subconjunto semi-algébrico de $\mathbb{C}_{p}^{M}$.

(1) $S$ e $\pi: \mathbb{C}_{p}^{M} \rightarrow \mathbb{C}_{p}^{M-1}$ é a projeção canônica nas $M-1$ primeiras variáveis, então $\pi(A)$ é um subconjunto semi-algébrico de $\mathbb{C}_{p}^{M-1}$.

(2) O fecho de $A, \bar{A} \subseteq \mathbb{C}_{p}^{M}$, é um subconjunto semi-algébrico de $\mathbb{C}_{p}^{M}$.

Prova. (i) Seja $\phi\left(X_{1}, \ldots, X_{M}\right)$ a $L_{a, P^{-}}^{\mathbb{C}_{p}}$ fórmula livre de quantificadores que define $A$. Então por 4.2 existe uma $L_{a, P^{-}}^{\mathbb{C}_{P}}$ fórmula livre de quantificadores $\psi\left(X_{1}, \ldots, X_{M-1}\right)$ tal que

$$
\mathbb{C}_{p} \models \exists X_{M} \phi\left(X_{1}, \ldots, X_{M}\right) \leftrightarrow \psi\left(X_{1}, \ldots, X_{M-1}\right)
$$

Como $\left(x_{1}, \ldots, x_{M-1}\right) \in \pi(A)$ se, e só se, existe $x_{M}$ tal que $\left(x_{1}, \ldots, x_{M-1}, x_{M}\right) \in A$, então $\psi$ define $\pi(A)$. Isto prova $(i)$. 
(ii) Seja $\phi$ como em (i). Observemos que

$$
\begin{aligned}
& \left(x_{1}, \ldots, x_{M}\right) \in \bar{A} \Longleftrightarrow \\
& \forall y\left[y=0 \vee \exists z_{1} \ldots z_{M}\left(\phi\left(z_{1}, \ldots, z_{M}\right) \wedge\left|x_{1}-z_{1}\right|<|y| \wedge \cdots \wedge\left|x_{M}-z_{M}\right|<|y|\right)\right] .
\end{aligned}
$$

A equivalência anterior e 4.2 provam (ii).

4.4 Analogamente, um subconjunto $A \subseteq \overline{\mathbb{Q}}_{p}^{M}$ é chamado semi-algébrico se é um elemento da álgebra de Boole gerada pelos conjuntos da forma

$$
\left\{x \in \overline{\mathbb{Q}}_{p}^{M}: f(x)=0,|g(x)| \leq|h(x)|\right\},
$$

onde $f, g, h \in \overline{\mathbb{Q}}_{p}\left[X_{1}, \ldots, X_{M}\right]$.

Seja $L_{a, P}^{\bar{Q}_{p}}$ a linguagem obtida ao agregar a $L_{a, P}$ um símbolo de constante por cada elemento de $\overline{\mathbb{Q}}_{p}$. De novo pelo Teorema 1.18, $\overline{\mathbb{Q}}_{p}$ possui eliminação de quantificadores na linguagem $L_{a, P}^{\overline{\mathbb{Q}}_{p}}$.

É claro que um subconjunto $A \subseteq \overline{\mathbb{Q}}_{p}^{M}$ é semi-algébrico se, e só se, $A$ é definido por uma $L_{a, P}^{\bar{Q}_{p}}$-fórmula livre de quantificadores, e então o seguinte lema se satisfaz.

Lema 4.5. Seja A um subconjunto semi-algébrico de $\overline{\mathbb{Q}}_{p}^{M}$.

(1) Se $\pi: \overline{\mathbb{Q}}_{p}^{M} \rightarrow \overline{\mathbb{Q}}_{p}^{M-1}$ é a projeção canônica, então $\pi(A)$ é um subconjunto semi-algébrico de $\overline{\mathbb{Q}}_{p}^{M-1}$.

(2) O fecho de $A, \bar{A} \subseteq \overline{\mathbb{Q}}_{p}^{M}$, é um subconjunto semi-algébrico de $\overline{\mathbb{Q}}_{p}^{M}$.

\section{Funções Analíticas p-Ádicas CoMplexas}

Nesta seção $F$ denota um corpo completo com respeito à norma dada por um valor absoluto não trivial $\mid$. 
4.6 Sejam $m \in \mathbb{N}, 0<r_{1}, \ldots, r_{m} \in \mathbb{R}$. O polidisco em $F^{m}$ com centro em $x=$ $\left(x_{1}, \ldots, x_{m}\right)$ e raio $r=\left(r_{1}, \ldots, r_{m}\right)$ é definido por

$$
\Delta(x, r):=\left\{\left(y_{1}, \ldots, y_{m}\right) \in F^{m}:\left|x_{1}-y_{1}\right|<r_{1}, \ldots,\left|x_{m}-y_{m}\right|<r_{m}\right\} .
$$

O polidisco fechado em $F^{m}$ com centro em $x=\left(x_{1}, \ldots, x_{m}\right)$ e raio $r=\left(r_{1}, \ldots, r_{m}\right)$ está dado por

$$
\bar{\Delta}(x, r):=\left\{\left(y_{1}, \ldots, y_{m}\right) \in F^{m}:\left|x_{1}-y_{1}\right| \leq r_{1}, \ldots,\left|x_{m}-y_{m}\right| \leq r_{m}\right\}
$$

Em particular, para $x=0$ temos as seguintes notações

$$
\Delta(r):=\Delta(0, r), \quad \bar{\Delta}(r)=\bar{\Delta}(0, r) .
$$

Também serão usadas as seguintes abreviações

$$
|x|<r \Longleftrightarrow\left|x_{j}\right|<r_{j}, j=1, \ldots, m . \quad|x| \leq r \Longleftrightarrow\left|x_{j}\right| \leq r_{j}, \quad j=1, \ldots, m .
$$

Seja $r^{\prime}=\left(r_{1}^{\prime}, \ldots, r_{m}^{\prime}\right), 0<r_{1}^{\prime}, \ldots, r_{m}^{\prime} \in \mathbb{R}$, então $r<r^{\prime}\left(\right.$ resp. $\left.r \leq r^{\prime}\right)$ denota $r_{j}<r_{j}^{\prime}$ (resp. $r_{j} \leq r_{j}^{\prime}$ ) para $j=1, \ldots, m$.

Finalmente lembramos que se $i=\left(i_{1}, \ldots, i_{m}\right) \in \mathbb{N}^{m}$, então $|i|=i_{1}+\cdots+i_{m}$.

Definição 4.7. Seja $f=\sum_{i \in \mathbb{N}^{m}} a_{i} X^{i} \in F \llbracket X_{1}, \ldots, X_{m} \rrbracket$, e $r=\left(r_{1}, \ldots, r_{m}\right), 0<r_{i} \in$ $\mathbb{R}$. A série $f$ é dita convergente em $\bar{\Delta}(r)$ se

$$
\sum_{i \in \mathbb{N}^{m}}\left|a_{i}\right| r^{i}<\infty
$$

A série $f$ é dita convergente em $\Delta(r)$ se $f$ converge em $\bar{\Delta}\left(r^{\prime}\right)$ para todo $r<r^{\prime}$. Finalmente $f$ é dita convergente se é convergente em $\Delta(r)$, para algum $r>0$.

Seja $f=\sum a_{i} X^{i}$ convergente em $\Delta(r)$. Para cada $x \in \Delta(r)$ a série $\sum a_{i} x^{i}$ converge absolutamente, e a soma $\widetilde{f}(x)$ é uma função contínua de $x$. O seguinte lema permite identificar uma série formal de potências com sua função associada $\tilde{f}$. 
Lema 4.8. (ver [Se, LG2.3]) $\tilde{f}=0$ implica $f=0$.

Definição 4.9. Seja $U \subseteq F^{n}$ um aberto e seja $\phi: U \rightarrow F$ uma função. Então $\phi$ é chamada analítica em $U$ se para cada $x \in U$ existe uma série de potências $f$ e um raio $r>0$ tal que

(1) $\Delta(x, r) \subseteq U, \mathrm{e}$

(2) $f$ converge em $\Delta(r)$, e para cada $h \in \Delta(r), \phi(x+h)=\tilde{f}(h)$.

Note que se $\phi$ é analítica em $U$ e $x \in U$, então a série $f$ da definição é única e chama-se a expansão local de $\phi$ em $x$.

Seja $U \subseteq F^{m}$ e $\phi=\left(\phi_{1}, \ldots, \phi_{n}\right): U \rightarrow F^{n}$. Então $\phi$ é dita analítica se $\phi_{i}$ é analítica para cada $1 \leq i \leq n$.

Teorema 4.10. (ver [Se, LG2.6]) Suponha $f: U \rightarrow V$ e $g: V \rightarrow W$ analíticas, onde $U \subseteq F^{m}, V \subseteq F^{s}, W \subseteq F^{n}$, são abertos. Então $g \circ f$ é analítica.

Definição 4.11. Quando $F=\mathbb{C}_{p}$, as funções analíticas são chamadas funções analíticas p-ádicas complexas.

\section{Variedades Analíticas $p$-Ádicas Complexas}

Como na seção anterior, $F$ denota um corpo completo com respeito à norma dada por um valor absoluto ||.

4.12 Seja $X$ um espaço topológico. Uma carta $c$ sobre $X$ é uma tripla $c=(U, \phi, n)$ tal que

(1) $U$ é um subconjunto aberto de $X$

(2) $n \in \mathbb{N}$ 
(3) $\phi: U \rightarrow \phi(U) \subseteq F^{n}$, onde $\phi(U)$ é um subconjunto aberto de $F^{n}$ e $\phi$ é um homeomorfismo.

4.13 Para uma carta $c=(U, \phi, n)$ sobre $X$, usaremos as seguintes notações

$$
\begin{array}{ll}
U=O(c)= & \text { Conjunto aberto de } c . \\
\phi=M(c)=\quad \text { Transformação de } c . \\
n=\operatorname{dim}_{F}(c)=\text { Dimensão de } c .
\end{array}
$$

4.14 Sejam $c=(U, \phi, n)$ e $d=(V, \psi, m)$ duas cartas sobre $X$. Dizemos que $c$ e $d$ são compatíveis se, para $W=U \cap V$, as transformações $\left.\psi \phi^{-1}\right|_{\phi(W)}$ e $\left.\phi \psi^{-1}\right|_{\psi(W)}$ são analíticas.

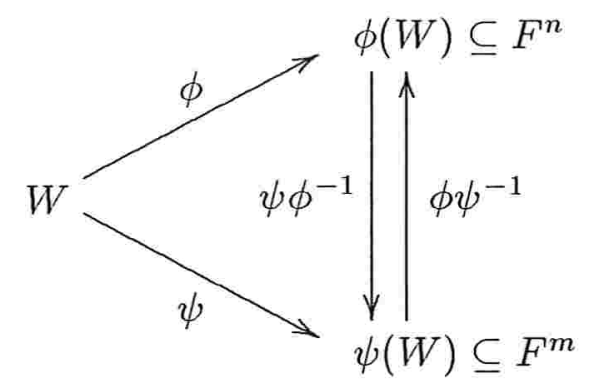

Se $c$ e $d$ são compatíveis, então $W \neq \varnothing$ implica $n=m$.

4.15 Uma família de cartas $\left\{c_{j}\right\}_{j \in J}$ sobre $X$ é chamada um recobrimento de $X$ se

$$
\bigcup_{j \in J} O\left(c_{j}\right)=X
$$

Um atlas $A$ sobre $X$ é uma família de cartas sobre $X$ a qual cobre $X$, e tal que as cartas da família são mutuamente compatíveis. 
Dois atlas $A$ e $A^{\prime}$ sobre $X$ são chamados compatíveis quando é satisfeita uma das duas condições equivalentes seguintes

(1) $A \cup A^{\prime}$ é um atlas.

(2) Se $c \in A$ e $c^{\prime} \in A^{\prime}$, então $c$ e $c^{\prime}$ são compatíveis.

A compatibilidade de atlas é uma relação de equivalência. De fato, as propriedades de simetria e reflexividade são óbvias pela definição. Agora sejam $A_{1}, A_{2}$, e $A_{3}$ atlas, tais que $A_{1}$ é compatível com $A_{2}$, e $A_{2}$ é compatível com $A_{3}$. Dados $c_{1} \in A_{1} \mathrm{e}$ $c_{3} \in A_{3}$, temos que provar que $c_{1}$ e $c_{3}$ são compatíveis. Seja $V=O\left(c_{1}\right) \cap O\left(c_{3}\right)$. Quando $V=\varnothing$, então $c_{1}$ e $c_{3}$ são trivialmente compatíveis. Suponha $V \neq \varnothing$, e seja $\phi_{1}=M\left(c_{1}\right)$ e $\phi_{3}=M\left(c_{3}\right)$. Por simetria basta provar que $\phi_{3} \phi_{1}^{-1}$ é analítica sobre $\phi_{1}(V)$. Para cada $x \in V$, provaremos que $\phi_{3} \phi_{1}^{-1}$ é analítica em $\phi_{1}(x)$. Escolha $c_{2}=(U, \phi, n) \in A_{2}$ tal que $x \in U$. Então

$$
\phi \phi_{1}^{-1}: \phi_{1}(U \cap V) \rightarrow \phi(U \cap V)
$$

é analítica em $\phi_{1}(x)$, e

$$
\phi_{3} \phi^{-1}: \phi(U \cap V) \rightarrow \phi_{3}(U \cap V)
$$

é analítica em $\phi(x)$. Portanto $\phi_{3} \phi_{1}^{-1}=\left(\phi_{3} \phi^{-1}\right)\left(\phi \phi_{1}^{-1}\right)$ é analítica em $\phi_{1}(x)$.

Definição 4.16. Seja $X$ um espaço topológico. Uma estrutura de variedade analítica sobre $X$ é uma classe de equivalência de atlas compatíveis sobre $X$.

Um atlas $A$ sobre $X$ é dito pleno se para cada carta $c$ sobre $X$, com $c$ compatível com todas as $c^{\prime} \in A$, se tem que $c \in A$.

Claramente, cada classe de equivalência de atlas sobre $X$ contém exatamente um atlas pleno. Pode-se então definir equivalentemente a noção de variedade analítica da seguinte maneira: uma estrutura de variedade analítica sobre $X$ é a escolha de um atlas pleno sobre $X$. 
No que se segue, $X$ denota um espaço topológico fixo com uma estrutura de variedade analítica. $A(X)$ denota seu atlas pleno. Por uma carta sobre $X$ entende-se uma carta de $A(X)$.

Seja $X$ uma variedade analítica e $x \in X$, então $\operatorname{dim}_{x} X$, a dimensão de $X$ em $x$, é definida como a dimensão de uma carta $c$ sobre $X$ tal que $x \in O(c)$. A função $x \mapsto \operatorname{dim}_{x} X$ é localmente constante; se esta função é constante, e igual a $n$, dizemos que $X$ é de dimensão constante $n$.

Para alguns corpos destacados $F$, as variedades analíticas de dimensão constante $n$, recebem nomes especiais, por exemplo,

variedade analítica real, quando $F=\mathbb{R}$.

variedade analítica complexa, quando $F=\mathbb{C}$.

variedade analítica $p$-ádica, quando $F=\mathbb{Q}_{p}$.

Definição 4.17. Seja $F=\mathbb{C}_{p}$. Uma variedade analítica de dimensão constante $n$ é chamada variedade analítica p-ádica complexa.

Seja $B$ um subconjunto de $X$. Dizemos que $B$ é uma bola se existe uma carta $c=(U, \phi, n)$ tal que $B \subseteq U$ e $\phi B$ é um polidisco da forma

$$
\left\{\left(y_{1}, \ldots, y_{n}\right) \in F^{n}:\left|x_{1}-y_{1}\right|<r, \ldots,\left|x_{n}-y_{n}\right|<r\right\}
$$

para algum $\left(x_{1}, \ldots, x_{n}\right) \in F^{n}$, onde $0<r \in \mathbb{R}$.

4.18 Enunciamos um par de propriedades topológicas para uma variedade analítica $X$ (ver [Se, (LG 3.3)]).

(1) Todo ponto $x \in X$ possui uma vizinhança $B$ que é uma bola. Em particular, $X$ é localmente um espaço métrico completo (portanto um espaço de Baire).

(2) Suponha que $F$ é localmente compacto. Então se $X$ é Hausdorff, $X$ é localmente compacto.

Como $\mathbb{C}_{p}$ não é localmente compacto (ver $\left.[G],\right)$, o anterior item (2) mostra uma 
importante diferença topológica das variedades de Hausdorff analíticas p-ádicas complexas (as quais não necessariamente são localmente compactas) com as variedades de Hausdorff analíticas $p$-ádicas (resp. reais, resp. complexas).

Sejam $X$ e $Y$ variedades analíticas. Uma função $f: X \rightarrow Y$ é chamada analítica se

(1) $f$ é contínua, e

(2) $f$ é localmente dada por funções analíticas, isto é, existem atlas $A$ de $X$ e $B$ de $Y$ tais que se $c=(U, \phi, n) \in A$ e $d=(V, \psi, m) \in B$, então para $W=U \cap f^{-1}(V)$, a composição

$$
\phi(W) \stackrel{\phi^{-1}}{\longrightarrow} W \stackrel{f}{\longrightarrow} V \stackrel{\psi}{\longrightarrow} \psi(V)
$$

é analítica.

Seja $X$ uma variedade e $U$ um aberto em $X$. Se $A(X)$ é o atlas de $X$, definimos

$$
A_{U}=\{c \in A: O(c) \subseteq U\} .
$$

Então $A_{U}$ define um atlas pleno sobre $U$. O espaço $U$ junto com seu atlas $A_{U}$ é chamado uma subvariedade aberta de $X$.

Agora $f: U \rightarrow F^{n}$ é chamada analítica se é uma transformação analítica entre as variedades $U$ e $F^{n}$.

\section{Conjuntos Subanalíticos De $\mathbb{C}_{p}$}

Nesta seção, $M$ denotará uma variedade analítica $p$-ádica complexa de Hausdorff. Escreveremos simplesmente " $M$ uma variedade".

Definição 4.19. Seja $M$ uma variedade.

(1) Um subconjunto $S \subseteq M$ é chamado semi-analítico em um ponto $x \in M$ se $x$ possui uma vizinhança $U$ em $M$ tal que $U \cap S$ é um elemento da álgebra de 
Boole gerada pelos conjuntos da forma

$$
\{y \in U: f(y)=0,|g(y)| \leq|h(y)|\}
$$

onde $f, g, h$ são funções analíticas sobre $U\left(f, g, h: U \rightarrow \mathbb{C}_{p}\right)$.

(2) Um subconjunto $S \subseteq M$ é chamado semi-analítico em $M$ se $S$ é semi-analítico em cada ponto de $M$.

(3) Um subconjunto $S \subseteq M$ é chamado subanalítico em um ponto $x \in M$ se existe uma vizinhança $U$ de $x$ em $M$ e um conjunto semi-analítico $S^{\prime}$ em $U \times \mathcal{D}^{N}$, para algum $N$, tal que $U \cap S=\pi\left(S^{\prime}\right)$, onde $\pi: U \times \mathcal{D}^{N} \rightarrow U$ é a projeção canônica.

(4) Um subconjunto $S \subseteq M$ é chamado subanalítico em $M$ se $S$ é subanalítico em cada ponto de $M$.

Claramente todo subconjunto de $\mathcal{D}^{M}$, definido por uma $L_{a n}$-fórmula livre de quantificadores, é semi-analítico.

Lema 4.20. Seja $S \subseteq \mathcal{D}^{M}$ definivel em $\mathcal{D}$ por uma $L_{a n}^{D}$-fórmula. Então $S$ é subanalítico em $\mathcal{D}^{M}$.

Prova. Seja $S \subseteq \mathcal{D}^{M}$ definível em $\mathcal{D}$ por uma $L_{a n}^{D}$-fórmula, então pelo Teorema 3.7 existe uma $L_{a n}^{D}$-fórmula livre de quantificadores $\phi\left(X_{1}, \ldots, X_{M}\right)$ que define $S$. Assim, pelo Lema 3.3 existe uma $L_{a n}$-fórmula livre de quantificadores $\phi_{m}\left(X_{1} \ldots, X_{M}, X_{M+1}\right.$, $\left.\ldots, X_{M+m}\right), m \in \mathbb{N}$, tal que

$$
\mathcal{D} \models \phi\left(X_{1}, \ldots, X_{M}\right) \longleftrightarrow \exists X_{M+1} \cdots X_{M+m}\left(\phi_{m}\left(X_{1} \ldots, X_{M}, X_{M+1}, \ldots, X_{M+m}\right)\right) .
$$

Portanto, se $S^{\prime}$ é o subconjunto de $\mathcal{D}^{M+m}$ definido por $\phi_{m}$, então $S^{\prime}$ é semi-analítico e $S=\pi\left(S^{\prime}\right)$, onde $\pi: \mathcal{D}^{M} \rightarrow \mathcal{D}^{M+m}$ é a projeção canônica. Isto prova que $S$ é subanalítico en $\mathcal{D}^{M}$.

Definição 4.21. Um subconjunto $S$ de $\mathcal{D}^{M}$ é chamado fortemente subanalítico se $S$ é definível em $\mathcal{D}$ por uma $L_{a n}^{D}$-fórmula. 
Lema 4.22. Sejam $f(X)=a_{0}+a_{1} X+\cdots+a_{n} X^{n}, g(X)=b_{0}+b_{1} X+\cdots+b_{m} X^{m}$ dois polinômios em $\mathbb{C}_{p}[X]\left(X=X_{1}\right)$. Suponha que existe $\alpha \in \mathcal{D}$ tal que $\alpha \neq 0$ e

$$
|f(\alpha)|=|g(\alpha)| \neq 0
$$

Então existem infinitos $\sigma \in \mathcal{D}$ tais que $|f(\sigma)|=|g(\sigma)|$.

Prova. Sem perda de generalidade podemos supor $a_{n} \neq 0, b_{m} \neq 0$. Tome $\beta \in \mathcal{D}$ com $0<|\beta|<|\alpha|$ tal que

$$
\left|a_{k}\left(\sum_{j=1}^{k}\left(\begin{array}{c}
k \\
j
\end{array}\right) \alpha^{k-j} \beta^{j}\right)\right|<|f(\alpha)|
$$

para todo $k=1, \ldots, n, \mathrm{e}$

$$
\left|b_{k}\left(\sum_{j=1}^{k}\left(\begin{array}{l}
k \\
j
\end{array}\right) \alpha^{k-j} \beta^{j}\right)\right|<|g(\alpha)|
$$

para todo $k=1, \ldots, m$. Assim

$$
\begin{aligned}
|f(\alpha+\beta)| & =\left|a_{0}+a_{1}(\alpha+\beta)+\cdots+a_{n}(\alpha+\beta)^{n}\right| \\
& =\left|a_{0}+\sum_{k=1}^{n} a_{k}(\alpha+\beta)^{k}\right| \\
& =\left|a_{0}+\sum_{k=1}^{n} a_{k}\left(\sum_{j=0}^{k}\left(\begin{array}{l}
k \\
j
\end{array}\right) \alpha^{k-j} \beta^{j}\right)\right| \\
& =\left|a_{0}+\sum_{k=1}^{n} a_{k}\left(\alpha^{k}+\sum_{j=1}^{k}\left(\begin{array}{c}
k \\
j
\end{array}\right) \alpha^{k-j} \beta^{j}\right)\right| \\
& =\left|a_{0}+\sum_{k=1}^{n} a_{k} \alpha^{k}+\sum_{k=1}^{n} a_{k}\left(\sum_{j=1}^{k}\left(\begin{array}{l}
k \\
j
\end{array}\right) \alpha^{k-j} \beta^{j}\right)\right| \\
& =\left|f(\alpha)+\sum_{k=1}^{n} a_{k}\left(\sum_{j=1}^{k}\left(\begin{array}{l}
k \\
j
\end{array}\right) \alpha^{k-j} \beta^{j}\right)\right|
\end{aligned}
$$


mas

$$
\left|\sum_{k=1}^{n} a_{k}\left(\sum_{j=1}^{k}\left(\begin{array}{l}
k \\
j
\end{array}\right) \alpha^{k-j} \beta^{j}\right)\right| \leq \max \left\{\left|a_{k}\left(\sum_{j=1}^{k}\left(\begin{array}{c}
k \\
j
\end{array}\right) \alpha^{k-j} \beta^{j}\right)\right|: k=1, \ldots, n\right\}<|f(\alpha)| .
$$

Portanto

$$
|f(\alpha+\beta)|=|f(\alpha)| .
$$

Similarmente, $|g(\alpha+\beta)|=|g(\alpha)|$. Seja $\sigma=\alpha+\beta$, então $|\sigma|=|\alpha+\beta|=|\alpha| \leq 1$ e $|f(\sigma)|=|f(\alpha)|=|g(\alpha)|=|g(\sigma)|$.

Corolário 4.23. Sejam $f(X), g(X) \in \mathbb{C}_{p}[X]$ como no lema anterior. Seja $d=$ $\max \{\operatorname{grau}(f), \operatorname{grau}(g)\}$. Suponha que existem diferentes $\alpha_{0}, \ldots, \alpha_{d+1} \in \mathcal{D}$ tais que $\left|f\left(\alpha_{i}\right)\right|=\left|g\left(\alpha_{i}\right)\right|$ para $i=0, \ldots, d+1$. Então existem infinitos $\beta \in \mathcal{D}$ tais que $|f(\beta)|=|g(\beta)|$.

Lema 4.24. Seja $S \subseteq \mathcal{D}^{M+N}$ fortemente subanalitico, então existe uma cota $A \in \mathbb{N}$ tal que para cada $x \in \mathcal{D}^{M}$ com fibra finita $S_{x}$ temos que $\operatorname{card}\left(S_{x}\right) \leq A$.

Prova. Seja $\phi(X, Y)$ uma $L_{a n}^{D}$-fórmula livre de quantificadores definindo $S$. Aplicando o Lema 3.3 a $\phi$ (ver também a nota depois do Lema 2.7) obtemos uma $L_{a n}$-fórmula livre de quantificadores $\phi^{*}\left(X, Y, Y_{N+1}, \ldots, Y_{N+n}\right)$ tal que $S$ é a imagem injetiva pela projeção canônica, $\left(x, y, y_{N+1}, \ldots, y_{N+n}\right) \mapsto(x, y)$, de $S^{*} \subseteq \mathcal{D}^{M+N+n}$, definido por $\phi^{*}$. Portanto card $S_{x}=\operatorname{card} S_{x}^{*}$, para cada $x \in \mathcal{D}^{M}$.

Assim, substituindo $S$ por $S^{*}$ podemos assumir sem perda de generalidade que $\phi$ é uma $L_{a n}$-fórmula, isto é, $D$ não ocorre em $\phi$.

Agora, é suficiente provar por indução sobre $N$ a seguinte afirmação:

$R(N)$ : Se $\phi(X, Y)$ é uma $L_{a n}^{D}$-fórmula livre de quantificadores na qual $D$ é aplicado só a termos que não envolvem $Y=\left(Y_{1}, \ldots, Y_{N}\right)$, então existe uma cota $A$ como é requerida para $S \subseteq \mathcal{D}^{M+N}$ definido por $\phi$.

Claramente $R(0)$ é válida, $\operatorname{com} A=1$. Seja $N>0$ e suponha $R(N-1)$ válido. Seja $\phi(X, Y)$ uma fórmula como foi estabelecida em $R(N)$. Para provar $R(N)$ para 
esta $\phi$ podemos substituir $D\left(t_{1}, t_{2}\right)$ em $\phi$ por novas $X$-variáveis e reduzir ao caso em que $\phi$ é uma $L_{a n}$-fórmula. Com $\phi$ desta forma podemos usar a prova do Lema 2.12 para ver que existe $L_{a n}^{D}$-fórmula livre de quantificadores $\phi^{d}(X, Z), Z=\left(Z_{1}, \ldots, Z_{N}\right)$ tal que

$$
\mathcal{D} \models \phi(X, Y) \leftrightarrow \phi^{d}(X, Z(Y))
$$

onde $Z(Y)=\left(Y_{1}-Y_{N}^{d^{N-1}}, \ldots, Y_{N-1}-Y_{N}^{d}, Y_{N}\right)$. Além disso, $Z_{N}$ aparece só polinomialmente em $\phi^{d}(X, Z)$.

Já que $y \mapsto z(y)$ é uma permutação de $\mathcal{D}^{N}$, é suficiente obter uma cota para a cardinalidade dos conjuntos finitos da forma

$$
S_{x}^{d}=\left\{z \in \mathcal{D}^{N}: \phi^{d}(x, z)\right\},
$$

onde $x$ percorre $\mathcal{D}^{N}$. Pelo Lema 3.6 sabemos que existe uma $L_{a n}^{D}$-fórmula livre de quantificadores $\psi\left(X, Z_{1}, \ldots, Z_{N-1}\right)$ na qual $D$ é aplicado só a termos que não envolvem $Z_{1}, \ldots, Z_{N-1}$ ) tal que

$$
\mathcal{D} \models \exists Z_{N} \phi^{d}(X, Z) \longleftrightarrow \psi\left(X, Z_{1}, \ldots, Z_{N-1}\right) .
$$

Agora aplicamos $R(N-1)$ a $\psi$ e obtemos uma cota $A_{1} \in \mathbb{N}$ tal que para todo $x \in \mathcal{D}^{M}$ temos

$$
\operatorname{card}\left\{\left(z_{1}, \ldots, z_{N-1}\right): \mathcal{D} \models \exists Z_{N} \phi^{d}\left(x, z_{1}, \ldots, z_{N-1}, z_{n}\right)\right\} \in\left\{0,1, \ldots, A_{1}\right\} \cup\{\infty\} .
$$

Afirmamos que existe uma cota $A_{2} \in \mathbb{N}$ tal que para todo $\left(x, z_{1}, \ldots, z_{N-1}\right) \in$ $\mathcal{D}^{M+(N-1)}$ temos

$$
\operatorname{card}\left\{z_{N}: \mathcal{D} \models \phi^{d}\left(x, z_{1}, \ldots, z_{N-1}, z_{N}\right)\right\} \in\left\{0,1, \ldots, A_{2}\right\} \cup\{\infty\} .
$$

É claro que $A=A_{1} A_{2}$ é uma cota para o cardinal de conjuntos finitos da forma $S_{x}^{d}$, $x \in \mathcal{D}^{M}$. Para ver que $A_{2}$ existe, notemos que, salvo equivalências, $\phi^{d}(X, Z)$ é uma disjunção de conjunções

$$
f_{0} \neq 0 \bigwedge_{1 \leq i \leq k} f_{i}=0 \bigwedge_{0 \leq j \leq l}\left|g_{j}\right| \leq\left|h_{j}\right| \bigwedge_{l+1 \leq j \leq l+s}\left|h_{j}\right|<\left|g_{j}\right|,
$$


e podemos assumir que $\phi^{d}$ é uma dessas conjunções.

Agora, $f_{i}, g_{j}$, e $h_{j}$ são polinomiais em $Z_{N}$, para $i=0, \ldots, k$, e $j=0, \ldots, l+s$. Sejam $E_{f_{i}}, E_{g_{j}}, E_{h_{j}}$, seus respectivos graus (na variável $Z_{N}$ ).

Finalmente

$$
A_{2}=2+\sum_{\substack{0 \leq i \leq k \\ 0 \leq j \leq l+s}} E_{f_{i}}+E_{g_{j}}+E_{h_{j}}
$$

é uma cota como foi requerida.

De fato, suponha que existem $z_{0}, \ldots, z_{A_{2}} \in \mathcal{D}$ tais que (1) se satisfaz para $\left(x, z_{1}, \ldots, z_{N-1}, z_{\mu}\right)$ para $0 \leq \mu \leq A_{2}$. Então para $1 \leq i \leq k, f_{i}\left(x, z_{1}, \ldots, z_{N-1}, z_{N}\right)=$ 0 para todo $z_{N} \in \mathcal{D}$. Podemos supor que $g_{j}\left(x, z_{1}, \ldots, z_{N-1}, Z_{N}\right)$ é um polinômio não nulo (na variável $Z_{N}$ ) para $0 \leq j \leq l$.

Existe $\operatorname{assim} z_{\alpha} \in\left\{z_{0}, \ldots, z_{A_{2}}\right\}$ tal que

(a) $z_{\alpha} \neq 0$

(b) $f_{0}\left(x, z_{1}, \ldots, z_{N-1}, z_{\alpha}\right) \neq 0$,

(c) $0<\left|g_{j}\left(x, z_{1}, \ldots, z_{N-1}, z_{\alpha}\right)\right| \leq\left|h_{j}\left(x, z_{1}, \ldots, z_{N-1}, z_{\alpha}\right)\right|$, para $0 \leq j \leq l$,

(d) $\left|h_{j}\left(x, z_{1}, \ldots, z_{N-1}, z_{\alpha}\right)\right|<\left|g_{j}\left(x, z_{1}, \ldots, z_{N-1}, z_{\alpha}\right)\right|$, para $l+1 \leq j \leq l+s$.

Usando a prova do Lema 4.22 temos que existem infinitos $z_{\beta} \in \mathcal{D} \operatorname{com} 0<\left|z_{\beta}\right|<$ $\left|z_{\alpha}\right|$ tais que

(i) $\left|f_{0}\left(x, z_{1}, \ldots, z_{N-1}, z_{\alpha}+z_{\beta}\right)\right|=\left|f_{0}\left(x, z_{1}, \ldots, z_{N-1}, z_{\alpha}\right)\right| \neq 0$,

(ii) $\left|g_{j}\left(x, z_{1}, \ldots, z_{N-1}, z_{\alpha}+z_{\beta}\right)\right|=\left|g_{j}\left(x, z_{1}, \ldots, z_{N-1}, z_{\alpha}\right)\right|$, para $0 \leq j \leq l$, e

(iii) $\left|h_{j}\left(x, z_{1}, \ldots, z_{N-1}, z_{\alpha}\right)\right|=\left|h_{j}\left(x, z_{1}, \ldots, z_{N-1}, z_{\alpha}+z_{\beta}\right)\right|$, para $0 \leq j \leq l$,

Isto finaliza a prova do Lema.

Nota. Em [L], Lipshitz apresenta um resultado similar ao lema anterior para corpos completos arbitrários com ultramétrica.

Finalizamos este capítulo observando que a noção de subconjunto subanalítico não 
é dada para subconjuntos de $\overline{\mathbb{Q}}_{p}$ (o qual não é completo). No entanto, uma prova análoga à do Lema 4.24 demonstra o seguinte

Lema 4.25. Seja $S \subseteq I^{M+N}$ definível por uma $L_{a n}^{D}$-fórmula, então existe uma cota $A \in \mathbb{N}$ tal que para cada $x \in I^{M}$ com fibra finita $S_{x}$ temos que card $\left(S_{x}\right) \leq A$. 



\section{CAPÍTULO 5}

\section{SISTEMAS DE WEIERSTRASS EM $\overline{\mathbb{Q}}_{p}$ E $\mathbb{C}_{p}$}

Iniciamos este capítulo apresentando a noção de Sistema de Weierstrass. Depois mostramos que se $\overline{\mathbb{Q}}_{p}\langle X\rangle$ é como em 2.2 , então a coleção de anéis $\left(\overline{\mathbb{Q}}_{p}\left\langle X_{(n)}\right\rangle\right)_{n \geq 0}$ constitui um sistema de Weierstrass sobre $\overline{\mathbb{Q}}_{p}$. Em $[A,(10.3)]$ prova-se que a coleção de séries de potências convergentes em uma vizinhança da origem constitui um sistema de Weierstrass sobre um corpo completo. Neste capítulo obtemos um resultado análogo para $\overline{\mathbb{Q}}_{p}$ (não completo).

Usando o Teorema de Divisão de Weierstrass não Arquimediano, adaptamos a noção de sistema de Weierstrass para o caso não arquimediano. Finalmente estabelecemos uma relação entre estas noções e os resultados dos Capítulos 2 e 3.

$\mathrm{Na}$ conclusão desta tese indicamos como estas técnicas poderiam ser adaptadas para provar a modelo completude de algumas estruturas. 


\section{Sistemas DE Weierstrass}

Definição 5.1. Seja $F$ um corpo. Um sistema de Weierstrass (ou um $W$-sistema) sobre $F$ é uma família de anéis $\left(F\left\lfloor X_{(n)}\right\rfloor\right)_{n \in \mathbb{N}}$, onde $X_{(n)}=\left(X_{1}, \cdots, X_{n}\right)$, satisfazendo as seguintes condições

W1 Para $n=0$ o anel é $F$. Para todo $n>0$ temos $F\left[X_{(n)}\right] \subseteq F\left\lfloor X_{(n)}\right\rfloor \subseteq$ $F \llbracket X_{(n)} \rrbracket$. Se $\sigma$ é uma permutação de $\{1,2, \cdots, n\}$ e $f\left(X_{(n)}\right) \in F\left\lfloor X_{(n)}\right\rfloor$, então $f\left(X_{\sigma(1)}, \cdots, X_{\sigma(n)}\right) \in F\left\lfloor X_{(n)}\right\rfloor$. Além disso, para $m \geq n, F\left\lfloor X_{(m)}\right\rfloor \cap$ $F \llbracket X_{(n)} \rrbracket=F\left\lfloor X_{(n)}\right\rfloor$.

W2 Se $f \in F\left\lfloor X_{(n)}\right\rfloor$ é uma unidade de $F \llbracket X_{(n)} \rrbracket$, então $f$ é uma unidade de $F\left\lfloor X_{(n)}\right\rfloor$.

W3 Se $f \in F\left\lfloor X_{(n)}, X_{n+1}\right\rfloor$ e $f\left(0, X_{n+1}\right) \in F \llbracket X_{n+1} \rrbracket$ é não nula de ordem $d$ (i.e $\left.f\left(0, X_{n+1}\right)=\sum_{i=d}^{\infty} c_{i} X_{n+1}^{i} \operatorname{com} c_{d} \neq 0\right)$, então para todo $g \in F\left\lfloor X_{(n+1)}\right\rfloor$, existe $Q \in F\left\lfloor X_{(n+1)}\right\rfloor$ e $R_{i} \in F\left\lfloor X_{(n)}\right\rfloor,(0 \leq i \leq d-1)$, tal que

$$
g=Q f+\sum_{i=1}^{d-1} R_{i} X_{n+1}^{i}
$$

5.2 Listamos agora algumas propriedades dos sistemas de Weierstrass. Para cada $n \in \mathbb{N}, F\left\lfloor X_{(n)}\right\rfloor$ é um anel que satisfaz as seguintes propriedades (ver [D-L, (1.3)]).

(1) $F\left\lfloor X_{(n)}\right\rfloor$ é local, com ideal maximal $I$ gerado por $X_{1}, \cdots, X_{n}$. O completado de $F\lfloor X\rfloor$ com respeito a $I$ é $F \llbracket X \rrbracket$. Se $g \in F\lfloor X\rfloor$, e $X_{i}$ divide $g$ em $F \llbracket X \rrbracket$ então $X_{i}$ divide $g$ em $F\lfloor X\rfloor(i=1, \cdots, n)$.

(2) (Substituição) Se $X=\left(X_{1}, \cdots, X_{n}\right), Y=\left(Y_{1}, \cdots, Y_{m}\right), f \in F\lfloor X, Y\rfloor \mathrm{e}$ $g_{1}, \cdots, g_{m} \in(X) F\left\lfloor X_{(n)}\right\rfloor$ (o ideal maximal de $F\left\lfloor X_{(n)}\right\rfloor$ ) então

$$
f\left(X, g_{1}(X) \cdots, g_{m}(X)\right) \in F\lfloor X\rfloor .
$$

(3) $F\left\lfloor X_{(n)}\right\rfloor$ é fechado para derivadas $\partial / \partial X_{i}, 1 \leq i \leq n$. 
(4) (Preparação de Weierstrass) Suponhamos que $f \in F\left\lfloor X_{(n+1)}\right\rfloor$ é regular de ordem $d$ em $X_{n+1}$. Então existe uma unidade $Q \in F\left\lfloor X_{(n+1)}\right\rfloor$, e existem $R_{0}, \cdots, R_{d-1} \in\left(X_{(n)}\right) F\left\lfloor X_{(n)}\right\rfloor$ tais que

$$
f=Q w, \quad \text { onde } w\left(X_{(n)}, X_{n+1}\right)=X_{n+1}^{d}+\sum_{i=0}^{d-1} R_{i}\left(X_{(n)}\right) X_{n+1}^{i} .
$$

Além disso, esta fatoração é única e $w$ é chamado o polinômio de Weierstrass de $f$.

(5) $F\left\lfloor X_{1}, \cdots, X_{n}\right\rfloor$ é noetheriano para cada $n$.

Teorema 5.3 (Aproximação de Artin). (ver [D-L,(1.1)]) Seja $\left(F\left\lfloor X_{(n)}\right\rfloor\right)_{n \in \mathbb{N}} u m$ $W$-sistema sobre $F, c \in \mathbb{N}, X=\left(X_{1}, \ldots, X_{n}\right), Y=\left(Y_{1}, \ldots, Y_{m}\right), f=\left(f_{1}, \cdots, f_{r}\right) \in$ $F\lfloor X, Y\rfloor^{m}$ e seja $\bar{y} \in(X) F \llbracket X \rrbracket$ satisfazendo $f(X, \bar{y})=0$. Então existe $y \in F\lfloor X\rfloor$ tal que $f(X, y)=0$ e $y \equiv \bar{y} \bmod (X)^{c}$.

Para $X=\left(X_{1}, \ldots, X_{n}\right)$, lembramos que

$$
\overline{\mathbb{Q}}_{p}\langle X\rangle=\left\{f \in \overline{\mathbb{Q}}_{p} \llbracket X \rrbracket: \exists\left[K: \mathbb{Q}_{p}\right]<\infty \wedge f \in K \llbracket X \rrbracket\right\},
$$

onde para $n=0$ o anel correspondente é $\overline{\mathbb{Q}}_{p}$.

Lema 5.4. A coleção de anéis $\left(\overline{\mathbb{Q}}_{p}\left\langle X_{1}, \ldots, X_{n}\right\rangle\right)_{n \in \mathbb{N}}$ é um sistema de Weierstrass sobre $\overline{\mathbb{Q}}_{p}$.

Prova. W1. É evidente que $\overline{\mathbb{Q}}_{p}[X] \subseteq \overline{\mathbb{Q}}_{p}\langle X\rangle \subseteq \overline{\mathbb{Q}}_{p} \llbracket X \rrbracket$. Se $f\left(X_{1}, \ldots, X_{n}\right) \in \overline{\mathbb{Q}}_{p}\langle X\rangle$, então existe $\left[K: \mathbb{Q}_{p}\right]<\infty$, tal que $f \in K \llbracket X \rrbracket$. Seja $\sigma$ uma permutação de $\{1, \ldots, n\}$; é claro que $f\left(X_{\sigma(1)}, \ldots, X_{\sigma(n)}\right) \in K \llbracket X \rrbracket$, e portanto $f\left(X_{\sigma(1)}, \ldots, X_{\sigma(n)}\right) \in \overline{\mathbb{Q}}_{p}\langle X\rangle$.

Suponhamos $m \geq n$. Se $f \in \overline{\mathbb{Q}}_{p} \llbracket X_{(n)} \rrbracket \cap \overline{\mathbb{Q}}_{p}\left\langle X_{(m)}\right\rangle$, então existe $\left[K: \mathbb{Q}_{p}\right]<\infty$ tal que $f \in K \llbracket X_{(m)} \rrbracket$. Como $f \in \overline{\mathbb{Q}}_{p} \llbracket X_{(n)} \rrbracket$, então $f \in K \llbracket X_{(n)} \rrbracket$. Assim $f \in \overline{\mathbb{Q}}_{p}\left\langle X_{(n)}\right\rangle$.

Agora se $f \in \overline{\mathbb{Q}}_{p}\left\langle X_{(n)}\right\rangle$, então existe $\left[K: \mathbb{Q}_{p}\right]<\infty$ tal que $f \in K \llbracket X_{(n)} \rrbracket$, e portanto $f \in K \llbracket X_{(m)} \rrbracket$. Assim $f \in \overline{\mathbb{Q}}_{p} \llbracket X_{(n)} \rrbracket \cap \overline{\mathbb{Q}}_{p}\left\langle X_{(m)}\right\rangle$. 
Provamos que para $m \geq n, \overline{\mathbb{Q}}_{p} \llbracket X_{(n)} \rrbracket \cap \overline{\mathbb{Q}}_{p}\left\langle X_{(m)}\right\rangle=\overline{\mathbb{Q}}_{p}\left\langle X_{(n)}\right\rangle$.

W2. Seja $f \in \overline{\mathbb{Q}}_{p}\langle X\rangle$ uma unidade de $\overline{\mathbb{Q}}_{p} \llbracket X \rrbracket$. Então existe uma extensão $\left[K: \mathbb{Q}_{p}\right]<\infty$ tal que $f \in K \llbracket X \rrbracket$, e $0 \neq f(0)=a_{0} \in K$, assim $f$ é uma unidade de $K \llbracket X \rrbracket$, e portanto uma unidade de $\overline{\mathbb{Q}}_{p}\langle X\rangle$.

W3. Para $X=X_{(M)}, Y=Y_{(1)}$, seja $f(X, Y) \in \overline{\mathbb{Q}}_{p}\langle X, Y\rangle$ tal que $f(0, Y)=$ $\sum_{i=d}^{\infty} c_{i} Y^{i}$ com $c_{d} \neq 0$. Então existe $\left[K: \mathbb{Q}_{p}\right]<\infty$ tal que $f \in K \llbracket X, Y \rrbracket$.

Dada $g \in \overline{\mathbb{Q}}_{p}\langle X, Y\rangle$, existe $\left[K_{1}: \mathbb{Q}_{p}\right]<\infty$, tal que $g \in K_{1} \llbracket X, Y \rrbracket$. Sem perda de generalidade podemos supor $K=K_{1}$. Como $K$ é completo para a norma $p$-ádica, podemos usar o Teorema de Divisão de Weierestrass dado por Abhyankar em [A, (3.10)]. Temos então que existem $Q \in K \llbracket X, Y \rrbracket$ e $R_{i}(X) \in K \llbracket X \rrbracket, 0 \leq i \leq d-1$ tais que

$$
g=Q f+\sum_{i=0}^{d-1} R_{i}(X) Y^{i}
$$

É claro que $Q \in \overline{\mathbb{Q}}_{p}\langle X, Y\rangle$ e $R_{i}(X) \in \overline{\mathbb{Q}}_{p}\langle X\rangle$, para $0 \leq i \leq d-1$. Isto prova W3.

5.5 Um sistema de Weierstrass $\left(F\left\lfloor X_{(m)}\right\rfloor\right)_{m \in \mathbb{N}}$ sobre $\overline{\mathbb{Q}}_{p}$ se diz convergente se cada $f \in F\left\lfloor X_{(m)}\right\rfloor$ converge sobre um polidisco

$$
\Delta_{r}:=\left\{x \in \overline{\mathbb{Q}}_{p}^{m}:\left|x_{1}\right|<r, \cdots,\left|x_{m}\right|<r\right\}, \quad 0<r \in \mathbb{R} .
$$

Notemos que se $a, x \in \Delta_{r}$, então $a+x \in \Delta_{r}$, e portanto a série

$$
f(a+X)=\sum \frac{\partial^{|i|} f}{\partial X^{i}}(a) \frac{X^{i}}{i !}
$$

é uma série convergente sobre $\Delta_{r}$ e também está em $F\lfloor X]$. Este fato não necessariamente se tem para Sistemas de Weierstrass sobre corpos como $\mathbb{R}$ ou $\mathbb{C}$, e precisa-se impor como uma condição adicional na definição de sistema convergente de Weierstrass (ver [vd Dries, (1.4)]). 
Lema 5.6. Seja $X=X_{(m)}, f(X)=\sum a_{i} X^{i} \in \overline{\mathbb{Q}}_{p}\langle X\rangle$ convergente em um polidisco $\Delta_{r}=\left\{\left(x_{1}, \cdots, x_{m}\right) \in \overline{\mathbb{Q}}_{p}^{m}:\left|x_{1}\right|<r, \ldots,\left|x_{m}\right|<r\right\}, 0<r \in \mathbb{R}$. Suponhamos que $f$ é uma unidade de $\overline{\mathbb{Q}}_{p}\langle X\rangle$, isto é, existe $g(X) \in \overline{\mathbb{Q}}_{p}\langle X\rangle$ tal que

$$
g(X) f(X)=1 .
$$

Então existe um polidisco $\Delta_{r_{1}}=\left\{\left(x_{1}, \cdots, x_{m}\right) \in \overline{\mathbb{Q}}_{p}^{m}:\left|x_{1}\right|<r_{1}, \ldots,\left|x_{m}\right|<r_{1}\right\}$, $0<r_{1} \in \mathbb{R}$, tal que $g(X)$ converge em $\Delta_{r_{1}}$.

Prova. Como $f$ é uma unidade de $\overline{\mathbb{Q}}_{p}\langle X\rangle$, temos que $f(0) \neq 0$. Logo existe um polidisco $\Delta_{r_{1}} \subseteq \overline{\mathbb{Q}}_{p}^{m}, 0<r_{1} \in \mathbb{R}$, tal que $f(x) \neq 0$ para todo $x \in \Delta_{r_{1}}$. Portanto

$$
g(x)=\frac{1}{f(x)} \quad \text { para todo } x \in \Delta_{r_{1}} .
$$

Assim $g(X)$ converge em $\Delta_{r_{1}}$.

Lema 5.7. (ver $[\mathrm{A},(10.3)]) \operatorname{Seja} X=X_{(m)}, Y=Y_{1},\left[K: \mathbb{Q}_{p}\right]<\infty, f(X, Y) \in$ $K \llbracket X, Y \rrbracket$ uma série convergente em um polidisco $\Delta_{r} \subseteq K^{m+1}, f$ regular de ordem $d$ em $Y$, isto é

$$
f(0, Y)=\sum_{i \geq d} c_{i} Y^{i} \quad c_{d} \neq 0 .
$$

Se $g(X, Y) \in \overline{\mathbb{Q}}_{p}\langle X, Y\rangle$ converge em um polidisco $\Delta_{r_{1}} \subseteq K^{m+1}$, então existem $q(X, Y) \in K \llbracket X, Y \rrbracket e r_{0}(X), \ldots, r_{d-1}(X) \in K \llbracket X \rrbracket$ tais que

$$
g(X, Y)=q(X, Y) f(X, Y)+\sum_{i=0}^{d-1} r_{i}(X) Y^{i},
$$

onde q converge em um polidisco $\Delta_{r_{2}} \subseteq K^{m+1}$ e $r_{i}(X)$ converge no polidisco $\Delta_{r_{2}} K^{m}$, para $i=0, \ldots, d-1$.

Para $X=\left(X_{1}, \ldots, X_{m}\right)$, definimos

$$
\overline{\mathbb{Q}}_{p}\langle\langle X\rangle\rangle=\left\{f \in \overline{\mathbb{Q}}_{p}\langle X\rangle: \quad f \text { converge em uma vizinhança da origem }\right\} .
$$

Com esta definição e os dois lemas anteriores temos o seguinte 
Lema 5.8. A coleção de anéis dada por $\left(\overline{\mathbb{Q}}_{p}\left\langle\left\langle X_{(m)}\right\rangle\right\rangle\right)_{m \in \mathbb{N}}$ é um sistema de Weierstrass sobre $\overline{\mathbb{Q}}_{p}$ convergente.

Prova. W1 verifica-se facilmente. W2 segue do Lema 5.5. Para provar W3 basta aplicar o Lema 5.7 e observar que se $X=X_{(m)},\left[K: \mathbb{Q}_{p}\right]<\infty$, e $f \in K \llbracket X \rrbracket$ converge em

$$
\bar{\Delta}_{r}:=\left\{x \in K^{m}:\left|x_{1}\right| \leq r, \cdots,\left|x_{m}\right| \leq r\right\}, \quad 0<r \in \mathbb{R}
$$

então $f$ converge em $\Delta_{r}=\left\{x \in \overline{\mathbb{Q}}_{p}^{m}:\left|x_{1}\right| \leq r, \cdots,\left|x_{m}\right| \leq r\right\} \subseteq \overline{\mathbb{Q}}_{p}^{m}$.

De fato, tomemos $x=\left(x_{1}, \ldots, x_{m}\right) \in K^{m}$ tal que $\left|x_{i}\right|=r$, para $i=1, \ldots, m$; como $f$ converge em $\bar{\Delta}_{r} \subseteq K^{m}$ então

$$
\left|a_{i} x^{i}\right|=\left|a_{i}\right| r^{|i|} \longrightarrow 0 \text { quando }|i| \longrightarrow \infty
$$

Seja $y=\left(y_{1}, \ldots, y_{m}\right) \in \Delta_{r} \subseteq \overline{\mathbb{Q}}_{p}^{m}$, assim $\left|y_{i}\right| \leq r$. Para alguma extensão finita $\left[K_{1}: \mathbb{Q}_{p}\right]<\infty$, temos que $y \in K_{1}^{m}$ e $f \in K_{1} \llbracket X \rrbracket$. Já que

$$
\left|a_{i} y^{i}\right|=\left|a_{i}\right|\left|y_{1}^{i_{1}}\right| \cdots\left|y_{m}^{i_{m}}\right| \leq\left|a_{i}\right| r^{i_{1}} \cdots r^{i_{m}}=\left|a_{i}\right| r^{|i|} \longrightarrow 0
$$

então, pela completude de $K_{1}, f$ converge em $y$.

\section{Sistemas de Weierstrass NÃo Arquimedianos}

Adotamos nesta seção a notação dada em 2.20. Isto é, seja $F$ um corpo com uma ultramétrica $\|$, e $X=\left(X_{1}, \ldots, X_{n}\right)$. Denotamos por $F\{X\}$ o subanel de $F \llbracket X \rrbracket$ que contém as séries $f=\sum_{i \in \mathbb{N}^{n}} a_{i} X^{i}$, tais que, $\left|a_{i}\right| \rightarrow 0$, quando $|i| \rightarrow \infty$. E para cada $f \in F\{X\}$, definimos $\|f\|=\max \left\{\left|a_{i}\right|: i \in \mathbb{N}^{n}\right\}$.

O anel $\{f \in F\{X\}:\|f\| \leq 1\}$ é denotado por $F^{\prime}\{X\}$; o ideal $\left\{f \in F^{\prime}\{X\}:\|f\|<1\right\}$ é denotado por $F^{\prime \prime}\{X\}$, e $\bar{F}\{X\}$ representa $F^{\prime}\{X\} / F^{\prime \prime}\{X\}$. Em particular $\bar{F}=$ $F^{\prime} / F^{\prime \prime}$ denota o corpo residual de $F$. 
A sobrejeção canônica $F^{\prime}\{X\} \rightarrow \bar{F}\{X\}$ é denotada por $f \rightarrow \bar{f}$. Seja $X=$ $\left(X_{1}, \ldots, X_{n}\right)$ e $Y=Y_{1}$. Um elemento $f \in F\{X, Y\}$, com $\|f\|=1$, se chama regular de grau d em $Y$ se $\bar{f} \in \bar{F}\{X, Y\}$ é da forma

$$
\lambda Y^{d}+\sum_{i<d} c_{i}(X) Y^{i}
$$

onde $\lambda \in \bar{F}, \lambda \neq 0$ e $c_{0}, \ldots, c_{d-1} \in \bar{F}[X]$.

Definição 5.9. Seja $F$ um corpo com ultramétrica. Um sistema de Weierstrass não arquimediano sobre $F$ é uma família de anéis $\left(F\left\lfloor\left\lfloor X_{(n)}\right\rfloor\right\rfloor\right)_{n \in \mathbb{N}}$, onde $X_{(n)}=$ $\left(X_{1}, \cdots, X_{n}\right)$, satisfazendo as seguintes condições

UW1 Para $n=0$ o anel é $F$. Para todo $n>0$ temos $F\left[X_{(n)}\right] \subseteq F\left\lfloor\left\lfloor X_{(n)}\right\rfloor \subseteq\right.$ $F\left\{X_{(n)}\right\}$. Se $\sigma$ é uma permutação de $\{1,2, \cdots, n\}$ e $f\left(X_{(n)}\right) \in F\left\lfloor X_{(n)}\right\rfloor$, então $f\left(X_{\sigma(1)}, \cdots, X_{\sigma(n)}\right) \in F\left\lfloor\left\lfloor X_{(n)}\right\rfloor\right\rfloor$. Além disso, para $\left.m \geq n, F\left\lfloor X_{(m)}\right\rfloor\right\rfloor \cap$ $F \llbracket X_{(n)} \rrbracket=F\left\lfloor\left\lfloor X_{(n)}\right\rfloor\right\rfloor$.

UW2 Se $\left.f \in F\left\lfloor X_{(n)}\right\rfloor\right\rfloor$ é uma unidade de $F\left\{X_{(n)}\right\}$, então $f$ é uma unidade de $F\left\lfloor\left\lfloor X_{(n)}\right\rfloor\right\rfloor$.

UW3 Se $f \in F\left\lfloor X_{(n)}, X_{n+1}\right\rfloor$ é regular de grau $d$ em $X_{n+1}$, então para todo $g \in$ $F\left\lfloor\left\lfloor X_{(n+1)}\right\rfloor\right\rfloor$, existe $\left.Q \in F\left\lfloor X_{(n+1)}\right\rfloor\right\rfloor$ e $\left.R_{i} \in F\left\lfloor X_{(n)}\right\rfloor\right\rfloor,(0 \leq i \leq d-1)$, tal que

$$
g=Q f+\sum_{i=1}^{d-1} R_{i} X_{n+1}^{i}
$$

Com esta definição e o Teorema 2.21 temos o seguinte

Lema 5.10. Seja F um corpo completo para uma ultramétrica. Então a coleção de anéis $\left(F\left\{X_{(n)}\right\}\right)_{n \geq 0}$ constitui um sistema de Weierstrass não arquimediano sobre $F$. 
Chamamos pré-sistema de Weierstrass não arquimediano sobre $F$ uma coleção de anéis $\left(F\left\lfloor\left\lfloor X_{(n)}\right\rfloor\right\rfloor\right)_{n \in \mathbb{N}}$ satisfazendo UW3, e tal que $F\left\lfloor X_{(n)}\right\rfloor \subseteq F\left\{X_{(n)}\right\}$ para todo $n \geq 0$.

Lembramos que $f \in F^{\prime}\{X\}$ se, e só se, $\|f\| \leq 1$. De novo pelo Teorema 2.21 temos o seguinte

Lema 5.11. Seja F um corpo completo para uma ultramétrica. Então a coleção de anéis $\left(F^{\prime}\left\{X_{(n)}\right\}\right)_{n \geq 0}$ constitui um pré-sistema de Weierstrass não arquimediano sobre $F$.

Lembramos que $I\{X\}$ é o subanel de $\overline{\mathbb{Q}}_{p}\{X\} \cap \overline{\mathbb{Q}}_{p}\langle X\rangle$ que contém as séries com coeficientes em $I$.

Lema 5.12. $\left(I\left\{X_{(n)}\right\}\right)_{n \geq 0}$ é um pré-sistema de Weierstrass não arquimediano sobre $\mathbb{C}_{p}$.

Prova. Claramente $I\{X\} \subseteq \mathbb{C}_{p}\{X\}$ para $X=\left(X_{1}, \ldots, X_{n}\right), n \geq 0$. Agora seja $f(X, Y) \in I\{X, Y\}$ regular de grau $d$ em $Y\left(Y=Y_{1}, X=\left(X_{1}, \ldots, X_{n}\right)\right)$, e seja $g(X, Y) \in I\{X, Y\}$. Como $f$ e $g$ estão em $\overline{\mathbb{Q}}_{p}\langle X, Y\rangle$, então existe $\left[K: \mathbb{Q}_{p}\right]<\infty$ tal que $f, g \in K \llbracket X, Y \rrbracket$, e portanto $f, g \in K^{\prime}\{X, Y\}$. Usando o lema anterior temos que existem $Q \in K^{\prime}\{X, Y\}$ e $R_{i} \in K^{\prime}\{X\},(0 \leq i \leq d-1)$, tais que

$$
g=Q f+\sum_{i=1}^{d-1} R_{i} X_{n+1}^{i}
$$

É claro que $Q \in I\{X, Y\}$, e $R_{i}(X) \in I\{X\}$, para $(0 \leq i \leq d-1)$.

Definição 5.13. Seja $W=\left(\mathbb{C}_{p}\left\lfloor\left\lfloor X_{(n)}\right\rfloor\right\rfloor\right)_{n \geq 0}$ um pré-sistema de Weierstrass não arquimediano contido em $\left(I\left\{X_{(n)}\right\}\right)_{n \geq 0}$. Definimos $L_{W}^{D}$ como a linguagem que contém um símbolo de operação $m$-ário $f$ para cada $f \in \mathbb{C}_{p}\left\lfloor\left\lfloor X_{(m)}\right\rfloor\right\rfloor, m \geq 0$, um símbolo de operação binário $D$, e um símbolo de relação binário $P$.

Com as interpretações usuais $I$, e $\mathcal{D}$ são $L_{W}^{D}$-estruturas. 
Lema 5.14. Seja $W=\left(\mathbb{C}_{p}\left\lfloor\left\lfloor X_{(n)}\right\rfloor\right\rfloor\right)_{n \geq 0}$ um pré-sistema de Weierstrass não arquimediano contido em $\left(I\left\{X_{(n)}\right\}\right)_{n \geq 0}$ satisfazendo

(1) $I\left[X_{(m)}\right] \subseteq \mathbb{C}_{p}\left\lfloor\left\lfloor X_{(m)}\right\rfloor\right\rfloor$, para todo $m \geq 0$. Sejam $X=X_{(m)}, Y=Y_{(n)}$, e $f \in \mathbb{C}_{p}\left\lfloor\lfloor X, Y\rfloor\right.$, então $f$ pode ser escrita da forma $f=\sum_{i \in \mathbb{N}^{n}} a_{i}(X) Y^{i}$, com $a_{i}(X) \in \mathbb{C}_{p}\lfloor\lfloor X\rfloor$, e existe $d \in \mathbb{N}$ tal que para todo $i$ com $|i| \geq d$,

$$
a_{i}=\sum_{|j|<d} b_{i, j} a_{j}, \quad \text { com } b_{i, j} \in \mathbb{C}_{p}\lfloor\lfloor\rfloor\rfloor, \quad\left\|b_{i, j}\right\|<1 / p
$$

Além disso os $b_{i, j}$ podem ser escolhidos, para cada $j$ fixo com $|j|<d$, tais que $\left\|b_{i, j}\right\| \longrightarrow 0$ quando $|i| \longrightarrow \infty$.

(2) Para $f(X, Y) \in \mathbb{C}_{p}\lfloor\lfloor, Y\rfloor\rfloor$ satisfazendo (1), e $j$ fixo com $|j|<d$, a série $\bar{f} \in \mathbb{C}_{p} \llbracket X, Y \rrbracket$ definida por

$$
\begin{aligned}
\bar{f}= & \left(\sum_{i \in A(j)} V_{i} Y^{i}\right)+Y^{j}+\left(\sum_{i \in B(j)} p V_{i} Y^{i}\right) \\
& +\sum_{|i| \geq d}\left\{\left(\sum_{k \in A(j)} b_{i, k} V_{k}\right)+b_{i, j}+\left(\sum_{k \in B(j)} b_{i, k} p V_{k}\right)\right\} Y^{i},
\end{aligned}
$$

está em $\mathbb{C}_{p}\lfloor\lfloor, V, Y\rfloor\rfloor$ (os conjuntos $A(j)$ e $B(j)$ são como em 2.24).

(3) Dada $f \in \mathbb{C}_{p}\left\lfloor\lfloor, V, Y\rfloor\right.$, então $T(f) \in \mathbb{C}_{p}\lfloor\lfloor, V, Z\rfloor$, onde $T: I\{X, V, Y\} \rightarrow$ $I\{X, V, Z\}$ é dada por

$$
T\left(Y_{\lambda}\right)=Z_{\lambda}+\left(Z_{N}\right)^{d^{N-\lambda}} \quad \text { para } 1 \leq \lambda<N, \quad T\left(Y_{N}\right)=Z_{N},
$$

e a transformação $T$ é um isomorfismo de $\mathbb{C}_{p}\lfloor\lfloor X, V\rfloor$-álgebras .

Então $I$ e $\mathcal{D}$ possuem eliminação de quantificadores na linguagem $L_{W}^{D}$.

A seguir, na conclusão desta tese, indicaremos como o anterior resultado poderia ser usado na possível prova da modelo completude de algumas estruturas. 



\section{CONCLUSÃO}

Para finalizar, apresentamos alguns problemas abertos relacionados com os resultados obtidos nesta tese. Propomos possíveis caminhos para resolvê-los baseados no trabalho de van den Dries [vd Dries].

P1. Provar a modelo completude da estrutura $(I,+,-, \cdot, 0,1, P, E(x))$, onde $E(x):=$ $\left.\exp \left(p^{2} x\right)\right)$.

Um caminho possível para provar P1 é o seguinte: construir um pré-sistema de Weierstrass não arquimediano sobre $\mathbb{C}_{p}$ contido em $\left(I\left\{X_{(n)}\right\}\right)_{n \geq 0}$ a partir de funções analíticas em $I$ existencialmente definíveis em $(I,+,-, \cdot, 0,1, P, E(x))$. Depois usar o Teorema 5.14. como em [vd Dries, §4].

P2. Provar a modelo completude da estrutura $(\mathcal{D},+,-, \cdot, 0,1, P, E(x))$.

$\mathrm{O}$ mesmo caminho sugerido para resolver $\mathrm{P} 1$ deve provar $\mathrm{P} 2$.

P3. Mostrar a modelo completude da estrutura $\left(\overline{\mathbb{Q}}_{p},+,-, \cdot, 0,1, P,\left.E(x)\right|_{I}\right)$.

Para obter P3, podemos tentar adaptar de novo [vd Dries, §4], usando a transformação $\tau: \overline{\mathbb{Q}}_{p} \rightarrow I$ dada por

$$
\tau(x)=\left\{\begin{array}{lll}
\frac{p x}{x+1} & \text { se }|x| \neq 1 \\
x & \text { se } & |x|=1
\end{array}\right.
$$

P4. Mostrar a modelo completude da estrutura $\left(\mathbb{C}_{p},+,-, \cdot, 0,1, P,\left.E(x)\right|_{I}\right)$.

Uma prova de P3 deve mostrar P4. 



\section{REFERÊNCIAS}

[A] S.S. Abhyankar, Local Analytic Geometry, Academic Press, New York, (1964)

[B] R. Bianconi, Model completeness results for elliptic and abelian functions, Ann. of Pure and Applied Logic 54, 121-136 (1991).

[B2] R. Bianconi, Some results in the model theory of analytic functions, Doctoral Thesis, Oxford (1990).

[D] J. Denef, The rationality of the Poincaré series associated to the $p$-adic points on a variety, Invent. Math. 77, no. 1, 1-23 (1984).

[D-L] J.Denef and L. Lipshitz, Ultraproducts and aproximation in local rings II, Math. Ann. 253 (1980).

[D-vd Dries] J. Denef and L. van den Dries. p-Adic and real subanalitic sets, Ann. of Math. 128, 79-138 (1988).

[vd Dries] L. van den Dries, On the elementary theory of restricted elementary finctions, Journal Symbolic Logic 53, 796-808 (1988).

[F-vd Put] J. Fresnel and M. van den Put, Géométrie Analytique Rigide et Applications, Progress in Math. vol. 18, Birkhäuser (1981).

[G] F. Gouvea, p-adic Numbers, Springer Verlag, Berlin (1993).

[K] W. Krull, Allgemeine Bewertungstheorie, J. Reine Angew. Math. 167, 160-196 (1932).

[L] L. Lipshitz, Isolated points on fibers of affinoid varieties, J. Reine Angew. Math. 384, 208-220 (1988). 
[M] A. Macintyre, On definable subsets of p-adic fields, Journal of Symbolic Logic, vol. 41, Num. 3, 605-610 (1976).

[M-McK-vdD] A. Macintyre, K. McKenna, L. van den Dries, Elimination of Quantifiers in Algebraic Structures, Advances in Mathemathics 47, 74-78 (1983).

[Mat 1] H. Matsumura, Commutative Algebra, Benjamin (1970).

[Mat 2] H. Matsumura, Commutative Ring Theory, Cambridge Universite Press (1986).

[R] A. Robinson, Complete Theories, North-Holland, Amsterdam, (1956).

[S] G. Sacks, Saturated Model Theory, Benjamin, New York (1972).

[Se] J-P. Serre. Lie Algebras and Lie Groups, Lectures given at Harvard University, Mathematics Lecture Notes Series, Benjamin, (1964).

[Sh] J. R. Shoenfield, A Theorem of Quantifier Elimination, Symposiam mathematica, vol. 5, 173-176 (1971).

[Z-S] O. Zariski, P. Samuel, Commutative algebra. I, II. Princeton: van Nostrand 1960. 